\title{
可见光波段稀土激光晶体的研究进展
}

\author{
李 纳 ${ }^{1}$, 刘 斌 $^{1}$, 施佼佼 ${ }^{1}$, 薛艳艳 ${ }^{1}$, 赵衡煜 ${ }^{1}$, \\ 施张丽 ${ }^{1}$, 侯文涛 ${ }^{1}$, 徐晓东 ${ }^{2}$, 徐 ${ }^{1}$
}

(1. 同济大学 先进微结构材料教育部重点实验室, 上海 200092; 2. 江苏师范大学 物理与电子工程学院, 徐州 221116)

摘 要: 可见光激光在数据存储、光通讯、激光显示、激光医疗、激光打印以及科学研究等领域具有非常重要的应 用价值。随着蓝光 LD 泵浦源的商用化, 直接葲浦稀土离子掺杂激光晶体实现可见光激光输出吸引了人们极大的研 究兴趣。目前, 可见光稀土离子主要集中在 $\mathrm{Pr}^{3+} 、 \mathrm{Dy}^{3+} 、 \mathrm{~Tb}^{3+}$ 和 $\mathrm{Sm}^{3+}$ 等。其中, $\mathrm{Pr}^{3+}$ 的研究较多, 发光波长涵盖面较 广, 发射波段覆盖蓝光、绿光、红光、橙光; $\mathrm{Dy}^{3+}$ 和 $\mathrm{Tb}^{3+}$ 因为能够发射黄光以填补 $\mathrm{Pr}^{3+}$ 的不足也吸引了广泛的研究; 此外, $\mathrm{Sm}^{3+}$ 和 $\mathrm{Eu}^{3+}$ 也是典型的可见波段稀土发光离子。本文综述了近几年可见波段稀土离子掺杂激光晶体的研究现 状, 主要以 $\mathrm{Pr}^{3+} 、 \mathrm{Dy}^{3+} 、 \mathrm{~Tb}^{3+}$ 和 $\mathrm{Sm}^{3+}$ 掺杂 $\mathrm{YAlO}_{3}(\mathrm{YAP}) 、 \mathrm{Mg}: \mathrm{SrAl}_{12} \mathrm{O}_{19}(\mathrm{SRA})$ 等晶体为研究对象, 总结了一套适合 $\operatorname{Pr}^{3+}$ 掺杂材料的判据, 对晶体生长、结构、热学性能、偏振光谱性能和激光性能进行了系统的研究。

关 键 词: 激光晶体; 可见光; 铝酸钇; 铝酸锶; 综述

中图分类号: TQ174 文献标识码: A

\section{Research Progress of Rare-earth Doped Laser Crystals in Visible Region}

\author{
LI Na ${ }^{1}$, LIU Bin ${ }^{1}$, SHI Jiao-Jiao ${ }^{1}$, XUE Yan-Yan ${ }^{1}$, ZHAO Heng-Yu ${ }^{1}$, \\ SHI Zhang-Li ${ }^{1}$, HOU Wen-Tao ${ }^{1}$, XU Xiao-Dong ${ }^{2}$, XU Jun ${ }^{1}$ \\ (1. Key Laboratory of Advanced Microstructure Materials Ministry of Education, Tongji University, Shanghai 200092, China; \\ 2. School of Physics and Electronic Engineering, Jiangsu Normal University, Xuzhou 221116, China)
}

\begin{abstract}
Visible laser has been widely used in data storage, optical communication, laser display, laser medical treatment, laser printing, and scientific research. With the development of commercial blue light LD, the direct pumping of rare-earth ions doped laser crystals have attracted a lot of interests. Currently, visible ions mainly concentrat on $\mathrm{Pr}^{3+}, \mathrm{Dy}^{3+}, \mathrm{Tb}^{3+}$, and $\mathrm{Sm}^{3+}$. Trivalent praseodymium $\left(\operatorname{Pr}^{3+}\right)$ is a famous rare-earth ion with extensive laser transitions in visible region such as blue, green, red and orange light. However, there is still a region in yellow emission which is not covered by $\mathrm{Pr}^{3+} . \mathrm{Dy}^{3+}$ and $\mathrm{Tb}^{3+}$ have attracted much attention because of their yellow laser transitions. In addition, $\mathrm{Sm}^{3+}$ and $\mathrm{Eu}^{3+}$ are also typical visible rare-earth ions. In this paper, we mainly reviewed properties of rare-earth ions doped laser crystals for visible lasers, especially $\mathrm{Pr}^{3+}, \mathrm{Dy}^{3+}, \mathrm{Tb}^{3+} \mathrm{and}^{3+} \mathrm{Sm}^{3+}-\mathrm{doped}$ $\mathrm{YAlO}_{3}$ (YAP), $\mathrm{SrAl}_{12} \mathrm{O}_{19}$ (SRA) crystals. A design criterion for $\mathrm{Pr}^{3+}$ doped oxide materials was summarized. The crystal growth, structure, thermal properties, polarization spectroscopic and laser characteristics were analyzed in detail.
\end{abstract}

Key words: laser crystal; visible laser; $\mathrm{YAlO}_{3}$ (YAP); $\mathrm{SrAl}_{12} \mathrm{O}_{19}(\mathrm{SRA})$; review

收稿日期: 2018-09-03; 收到修改稿日期：2018-10-10

基金项目：国家自然科学基金(61621001, 51672190); 国家重点研发计划项目(2016YFB0701002, 2016YFB0402101) National Natural Science Foundation of China (61621001, 51672190); National Key Research and Development Program of China (2016YFB0701002, 2016YFB0402101)

作者简介: 李 纳(1992-), 女, 博士. E-mail: 1710069@tongji.edu.cn

通讯作者: 徐晓东, 教授. E-mail: xdxu79@jsnu.edu.cn; 徐 军, 教授. E-mail: xujun@mail.shcnc.ac.cn 
随着信息化时代的发展, 人们对特殊波段激光 的关注越来越多, 促使各国学者的研究重心往新波 段优良激光器的方向转变, 比如可见光波段和中红 外波段。其中, 可见光波段(380 780 nm)激光不仅在 人们日常生活中有广泛的应用(比如激光头灯、激光 提示器、医学、投影仪以及数据存储方面), 并且也 能应用到其他很多领域(比如新一代显示技术、显微 镜、可见光通讯、高端材料制备以及科研等)。2014 年, 诺贝尔物理学奖授予大功率蓝光 LED 的突破, 很大程度上反应了可见光激光在未来发展的重要 性。诺贝尔物理学奖的成果很大程度上推动了蓝光 $\mathrm{InGaN}$ 和 $\mathrm{GaN}$ 激光二极管的商品化。

大多数固体可见光波段激光主要由以下方式来 实现 ${ }^{[1-3]}$ : (1) 由非线性光学晶体的倍频或自倍频方 式获得, 把近红外波段激光转换为可见光激光输 出。2014 年, Zhao 等 ${ }^{[4]}$ 采用 $\mathrm{LD}$ 泵浦 $\mathrm{Nd}: \mathrm{YVO}_{4}$ 产 生红外光, 再使用 $\mathrm{LBO}$ 晶体腔内倍频蓝光 $(457 \mathrm{~nm})$, 输出功率达到 $21.8 \mathrm{~W}$, 光转换效率为 $36.3 \%$; 2014 年, Kantola 等 ${ }^{[5]}$ 使用 LBO 晶体腔内倍频黄光 $(588 \mathrm{~nm})$, 输 出功率达到 $20 \mathrm{~W}$, 并已成功用于钠信标激光器。山 东大学张怀金课题组 ${ }^{[6]}$ 在 $\mathrm{Nd}: \mathrm{GdCOB}$ 晶体中自倍 频实现 $3.01 \mathrm{~W}$ 的 $545 \mathrm{~nm}$ 绿色激光输出; 2016 年, 他 们又在 $\mathrm{Yb}$ : YCOB 晶体中自倍频实现 $570 \mathrm{~nm}$ 黄光 激光运转, 输出功率达到 $1.08 \mathrm{~W}^{[7]}$ 。(2)上转换激光 器方式获得。相比于非线性倍频技术, 此方式更简 单、直接, 且对材料物理性能和洜浦源的要求较低。 科研人员对可见光波段的上转换有大量研究。比如, Paschotta 等 ${ }^{[8]}$ 利用 $\mathrm{Nd}$ : YAG 激光器泵浦 $\mathrm{Tm}^{3+}$ 掺杂 氟化物光纤, 成功实现上转换蓝光 (480 nm)波段连 续激光运转, 输出功率达到 $230 \mathrm{~mW}$; Sandrock 等 ${ }^{[9]}$ 使用钛宝石泵浦 $\mathrm{Pr}, \mathrm{Yb}: \mathrm{ZBLAN}$ 光纤, 实现上转换 $635 \mathrm{~nm}$ 红光连续激光输出, 最大输出功率为 $1020 \mathrm{~mW}$, 光转换效率达到 $19 \%$ 。(3)通过二极管 LD 原浦激活 离子(比如过渡金属离子和稀土离子)掺杂激光材料 直接实现可见波段的激光运转。过渡金属离子在可 见波段和 3 5 $\mu \mathrm{m}$ 中红外领域有着明显优势。2009 年, 首次使用 $\mathrm{GaN} \mathrm{LD}$ 泵浦钛宝石, 实现红光波段 $19 \mathrm{~mW}$ 连续激光输出 ${ }^{[10]} ; 2015$ 年, Gürel 等 ${ }^{[11]}$ 使用 $\mathrm{LD}$ 泵浦钛宝石实现红光波段 $650 \mathrm{~mW}$ 连续激光输 出, 光转换效率为 $21.6 \%$, 并且获得平均输出功率 $450 \mathrm{~mW}$, 脉冲宽度 $58 \mathrm{fs}$ 的锁模激光运转; 虽然可 见波段激光的研究价值已被人们公认, 但是直接泵 浦稀土离子掺杂激光增益介质的可见光激光报道却 并不多。

高效蓝光泵浦源的出现(如 $\mathrm{InGaN}^{[12]}$ 激光二极 管和倍频光䈋浦半导体激光器 $(2 \omega-\mathrm{OPSL})^{[13-14]}$ )极大
地推动了稀土离子掺杂介质可见光波段激光输出的 复苏。目前, $\mathrm{GaN}$ 半导体二极管激光在 $390 \mathrm{~nm}$ 蓝光 附近的输出功率已经达到瓦级。 InGaN 半导体二极 管激光发射范围可以从 $390 \mathrm{~nm}$ 延伸到 $460 \mathrm{~nm}$, 甚 至可以达到更长的波段范围。尤其在 405 和 $450 \mathrm{~nm}$ 附 近的蓝光波段已经可以用于激光显示的实际应用, 其功率可以很容易达到 $3.5 \mathrm{~W}$, 而且价格较低。随着 高质量、大尺寸 $\mathrm{InGaN}$ 薄膜材料的生长, 会进一步 提升激光材料的输出功率。目前的泵浦功率可以通 过偏振耦合达到 $10 \mathrm{~W}$ 量级 ${ }^{[15]}$ 。进一步激光输出功 率的提升可以通过把单个二极管组合为二极管模组 的形式来实现。 $2 \omega$-OPSLs 是基于输出波长为 $1 \mu \mathrm{m}$ 附近的激光材料来实现激光输出的。目前这种方法, 已经获得了输出功率大于 $10 \mathrm{~W}$ 的绿光激光和输出 功率大于 $5 \mathrm{~W}$ 的 470 490 nm 波段的蓝光激光, 并且 光束质量良好, 带宽较窄, 是现今应用比较广泛的 泵浦源之一。此外, 随着 $\mathrm{AlGaN}$ 与 $\mathrm{InGaN}$ 半导体激 光光源的改进，其发射波长已延伸到紫外和更长波 段。本文将对稀土离子在可见光波段的研究现状进 行综述及分析。

理论上大多数的稀土离子在蓝光䈋浦下都能发 射可见光, 然而 13 个稀土离子中, $\mathrm{Ce} 、 \mathrm{Gd}$ 和 $\mathrm{Yb}$ 无 可见波段的激光跃迁。 $\mathrm{Pm}$ 存在可见波段激光跃迁， 但由于稳定同位素的缺乏, 实际应用很少。 $\mathrm{Nd}$ 和 $\mathrm{Tm}$ 存在大量的可见波段激光跃迁，但鲜有直接使 用蓝光半导体二极管激发 $\mathrm{Nd}$ 和 $\mathrm{Tm}$ 掺杂激光晶体 的报道。国内外直接发射可见光激光的研究大部分 集中于镨 $(\mathrm{Pr}) 、$ 镝 $(\mathrm{Dy}) 、$ 铽 $(\mathrm{Tb}) 、$ 钐 $(\mathrm{Sm})$ 、钬 $(\mathrm{Ho})$ 、 铒 $(\mathrm{Er}) 、$ 铕 $(\mathrm{Eu})$ 等几种稀土离子, 能级及能级跃迁如 图 1 所示。

\section{1 掺 $\mathbf{P r}^{3+}$ 激光晶体}

\section{1 掺 $\operatorname{Pr}^{3+}$ 激光晶体的光谱特性}

三价 $\mathrm{Pr}^{3+}$ 是应用较多的稀土离子。在 445、468 和 $486 \mathrm{~nm}$ 处, $\mathrm{Pr}^{3+}$ 吸收截面达到 $10^{-19} \mathrm{~cm}^{2}$ 量级, ${ }^{3} \mathrm{P}_{0}$ 上能级的苂光寿命约为几十微秒。其中 $445 \mathrm{~nm}$ 处的吸 收峰与 InGaN 激光二极管洜浦源的发射波长非常吻 合, $468 \mathrm{~nm}$ 处的吸收峰与 $2 \omega-$ OPSLs 原浦源的发射 波长非常吻合。相比于其他的稀土离子, $\operatorname{Pr}^{3+}$ 拥有大 量的辐射跃迁, 发光范围几乎覆盖了可见光波段的 红光、橙光、绿光、蓝光。因此, $\operatorname{Pr}^{3+}$ 掺杂激光材料 是目前最有潜力的可见波段激光材料。在蓝光波段 内, 存在一条强的 ${ }^{3} \mathrm{P}_{0}$ 到基态 ${ }^{3} \mathrm{H}_{4}$ 的发射跃迁, 由于 寿命较短且存在强的重吸收, 几乎无法实现有效激光 输出。如果能级䢃裂足够强, 截面在 $10^{-21} \mathrm{~cm}^{2}$ 


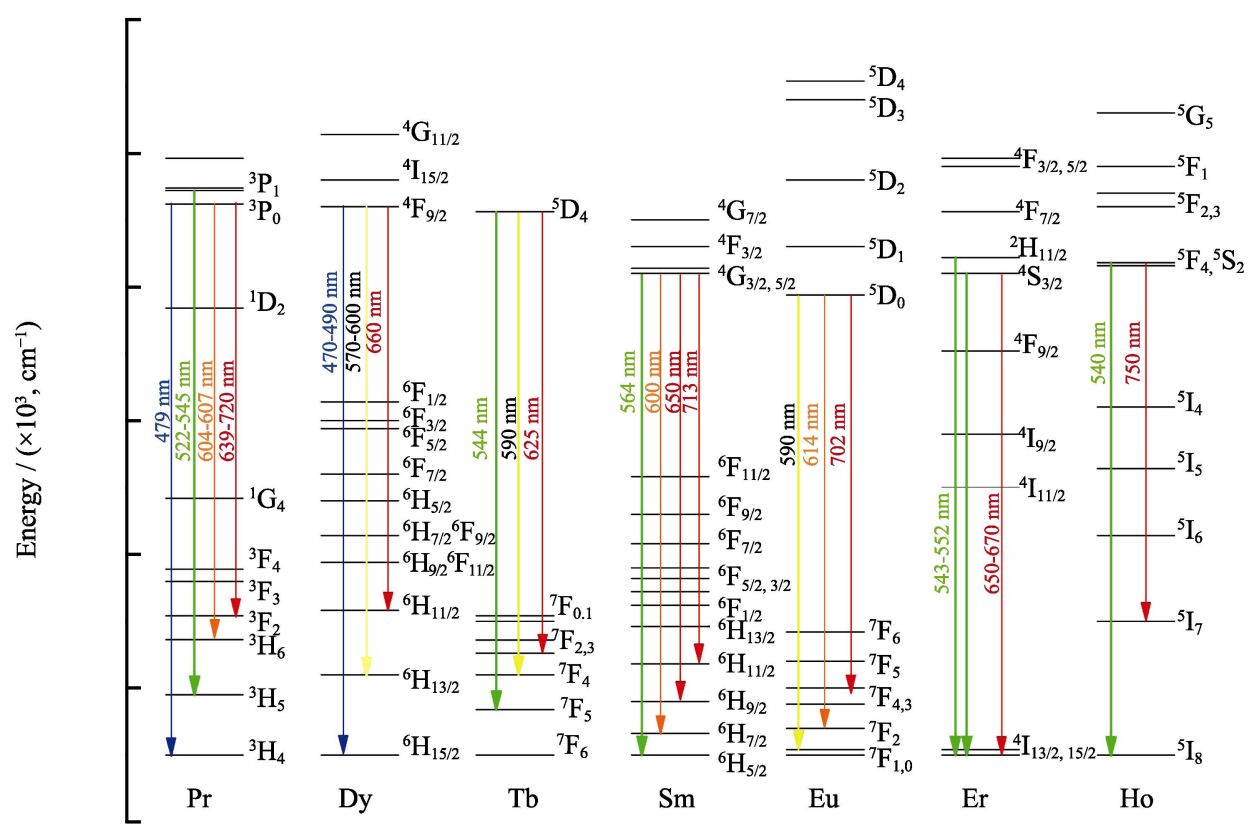

图 1 可见波段稀土离子(YLF 基质晶体)的能级跃迁图

Fig. 1 Energy levels of visible rare-earth ions in YLF crystal

到 $10^{-20} \mathrm{~cm}^{2}$ 的蓝光波段发光峰会形成准三能级激光 跃迁 ${ }^{[16]}$ 。短波长处四能级激光跃迁允许绿光波段 $520 \sim 550 \mathrm{~nm}$ 的激光运转。橙光波段的发射峰很强, 但 ${ }^{3} \mathrm{H}_{4} \rightarrow{ }^{1} \mathrm{D}_{2}$ 处存在重吸收。虽然属于禁戒跃迁, 但 $\mathrm{Pr}^{3+}$ 在 $\mathrm{LiYF}_{4}{ }^{[17]}$ 中对应吸收截面高达 $3 \times 10^{-20} \mathrm{~cm}^{2}$ 。 即使在波长大于 $600 \mathrm{~nm}$ 处, 低的重吸收 $10^{-21} \mathrm{~cm}^{2}$ 仍然对橙光激光产生强烈的影响, 尤其是在输出耦 合镜低透过率情况下，低的吸收损失会因为声子上 百次的腔内共振而影响较大。 $\operatorname{Pr}^{3+}$ 的主要发射是 $644 \mathrm{~nm}$ 红光, 对应的发射截面达 $10^{-19} \mathrm{~cm}^{2}$ 。在 695 和 $725 \mathrm{~nm}$ 附近发光峰的发射截面稍弱。两者都有宽的声子带 边, 且发射截面处于同一个量级, 可以用于宽光谱 调谐激光。

Pr : YAP 晶体室温偏振吸收光谱如图 2 所示。 可以发现, 三个偏振方向对应的吸收峰差异较大, 具有很强的偏振相关性。吸收光谱主要由 8 个吸收 带组成, 吸收对应的不同峰值主要由晶格场引起的 能级䢃裂造成。 ${ }^{1} \mathrm{G}_{4}$ 能级对应的吸收峰很难清晰地 分辨出来, 这主要是因为基态 ${ }^{3} \mathrm{H}_{4}$ 子能级的热致粒 子数堆积所引起背底噪声的影响。另外一些吸收带 难以从相邻吸收带中分离出来, 这是因为能级间的 相互作用, 强场耦合使得能级自身分裂低于相邻能 级的能级分裂。 $a 、 b$ 和 $c$ 偏振对应的吸收峰分别位 于 449、448 和 $449 \mathrm{~nm}$, 吸收截面分别为 $1.69 \times 10^{-20}$ 、 $3.41 \times 10^{-20}$ 和 $5.64 \times 10^{-20} \mathrm{~cm}^{2}$, FWHM 分别为 6.8 、5.2 和 $5.6 \mathrm{~nm}$ 。 $\mathrm{Pr}$ : YAP 晶体的吸收截面高于 $\mathrm{Pr}, \mathrm{Mg}: \mathrm{CaAl}_{12} \mathrm{O}_{19}\left(1.1 \times 10^{-20} \mathrm{~cm}^{2}\right)^{[19]}$ 和 $\mathrm{Pr}, \mathrm{Mg}: \mathrm{SrAl}_{12} \mathrm{O}_{19}$ $\left(1.3 \times 10^{-20} \mathrm{~cm}^{2}\right)^{[20]}$, 说明 $\mathrm{Pr}$ : YAP 晶体可以更高效地 使用 $\mathrm{GaN} / \mathrm{InGaN}$ LD 进行泵浦。

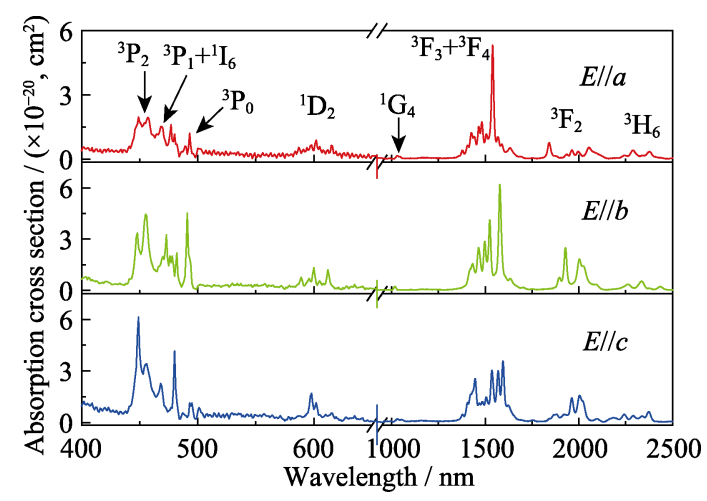

图 $2 \mathrm{Pr}$ : YAP 晶体的偏振吸收光谱 ${ }^{[18]}$

Fig. 2 Polarized absorption spectra of Pr : YAP crystal ${ }^{[18]}$

室温下 Pr：YAP 的偏振荧光光谱如图 3 所示。 从图中可以看到, 对应于三个偏振方向的发射强度 差距较大, 对偏振呈现出强烈的依赖性。这主要是 由于 YAP 正交结构晶体的各向异性 ${ }^{[21]}$ 。发射截面作 为预测材料激光性能非常重要的参数, 可通过 F-L 公式(1) ${ }^{[22]}$ 计算获得:

$$
\sigma_{\mathrm{em}}(\lambda)_{J J^{\prime}}=\frac{\lambda^{5}}{8 \pi n^{2} c \tau_{\mathrm{rad}}} \frac{I(\lambda)}{\int \lambda I(\lambda) \mathrm{d} \lambda}
$$

$I(\lambda)$ 代表测试的苂光强度, $A\left(J, J^{\prime}\right)$ 代表对应发射跃迁 的自发辐射几率。最强的发射截面为位于 $621 \mathrm{~nm}$ 的 ${ }^{3} \mathrm{P}_{0}$ $\rightarrow{ }^{3} \mathrm{H}_{6}$ 跃迁。其中 $b$ 偏振在 $621 \mathrm{~nm}$ 的发射截面值最 大, 为 $25.01 \times 10^{-20} \mathrm{~cm}^{2}$ 。大的发射截面表明 Pr: YAP 晶体的 ${ }^{3} \mathrm{P}_{0} \rightarrow{ }^{3} \mathrm{H}_{6}$ 跃迁很有可能获得高效率激光 输出。

表 1 总结了 $\operatorname{Pr}$ : YAP、Pr: SRA 和其他已实现 $\operatorname{Pr}^{3+}$ 激光输出晶体的吸收波长、吸收截面、吸收带 
宽、发射波长、发射截面、发射带宽以及 ${ }^{3} \mathrm{P}_{0}$ 能级 的荧光寿命参数。对于 ${ }^{3} \mathrm{H}_{4} \rightarrow{ }^{3} \mathrm{P}_{2}$ 吸收跃迁, $\mathrm{Pr}$ : YAP 和 $\operatorname{Pr}$ : SRA 对应的带宽较大, 适合使用蓝光 LD 原 浦进行洜浦。对于 ${ }^{3} \mathrm{P}_{1} \rightarrow{ }^{3} \mathrm{H}_{5}$ 跃迁, Pr: YAP 对应的发 射截面及参数 $\sigma_{\mathrm{em}} \tau$ 最大, 参数 $\sigma_{\mathrm{em}} \tau$ 与激光國值成反 比, 说明此通道 Pr：YAP 激光阈值更低, 性能更优, 有希望成为高效率激光通道。对于 ${ }^{3} \mathrm{P}_{0} \rightarrow{ }^{3} \mathrm{H}_{6}$ 跃迁, $\mathrm{Pr}$ ：YAP 对应的发射截面 $\sigma_{\mathrm{em}}$ 更大, 计算得到 ${ }^{3} \mathrm{P}_{0} \rightarrow$ ${ }^{3} \mathrm{H}_{6}$ 跃迁的 $\sigma_{\mathrm{em}} \tau$ 与 $\mathrm{Pr}: \mathrm{LiLuF}_{4}$ 和 $\mathrm{Pr}: \mathrm{LiYF}_{4}$ 接近 ${ }^{[24]}$, 明显高于 $\mathrm{Pr}, \mathrm{Mg}: \mathrm{SrAl}_{12} \mathrm{O}_{19}$ 晶体。因此, $\mathrm{Pr}$ : YAP 晶 体中 ${ }^{3} \mathrm{P}_{0} \rightarrow{ }^{3} \mathrm{H}_{6}$ 跃迁的激光阈值接近于 $\mathrm{Pr}: \mathrm{LiLuF}_{4}$ 和 $\mathrm{Pr}: \mathrm{LiYF}_{4}$ ，小于 $\mathrm{Pr}, \mathrm{Mg}: \mathrm{SrAl}_{12} \mathrm{O}_{19}$ 晶体。对于 ${ }^{3} \mathrm{P}_{0}$ $\rightarrow{ }^{3} \mathrm{~F}_{2}$ 跃迁, Pr: SRA 的发射截面 $\sigma_{\mathrm{em}}$ 大于 $\mathrm{Pr}$ : YAP, 其小于 $\operatorname{Pr}: \mathrm{LiLuF}_{4}$ 和 $\mathrm{Pr}: \mathrm{LiYF}_{4}$ 晶体, 发射截面与寿 命乘积 $\sigma_{\mathrm{em}} \tau$ 大于 $\operatorname{Pr}: \mathrm{YAP}$, 小于 $\mathrm{Pr}: \mathrm{LiLuF}_{4}$ 和 $\operatorname{Pr}: \mathrm{LiYF}_{4}$, 说明 $\mathrm{Pr}: \mathrm{SRA}$ 晶体中 ${ }^{3} \mathrm{P}_{0} \rightarrow{ }^{3} \mathrm{~F}_{2}$ 跃迁的阈 值小于 Pr:YAP, 大于 Pr: $\mathrm{LiLuF}_{4}$ 和 $\operatorname{Pr}: \mathrm{LiYF}_{4}$ 。虽 然激光阈值要大于氟化物, 但是 Pr: SRA 晶体的吸 收带宽是氟化物的 5 倍多, 这在很大程度上降低了 激光泵浦的难度。此外, 氟化物晶体的热机械性能 相对较低, 而 Pr: YAP、Pr: SRA 晶体的热机械性能
与 $\mathrm{YAG}$ 晶体相近, 优异的热学性能和机械强度有 利于激光器运转的稳定性。从上表中还可发现 $\operatorname{Pr}$ : SRA 的吸收和各发射跃迁带宽较大, 有利于可 调谐激光运转。综上所述, $\mathrm{Pr}$ : YAP 晶体的 ${ }^{3} \mathrm{P}_{1} \rightarrow{ }^{3} \mathrm{H}_{5}$ 、 ${ }^{3} \mathrm{P}_{0} \rightarrow{ }^{3} \mathrm{H}_{6} 、{ }^{3} \mathrm{P}_{0} \rightarrow{ }^{3} \mathrm{~F}_{4}$ 跃迁, Pr: SRA 晶体的 ${ }^{3} \mathrm{P}_{0} \rightarrow{ }^{3} \mathrm{~F}_{2}$ 跃迁对于高效连续激光运转具有很高的研究价值, Pr：SRA 晶体在可调谐、超快激光方面是非常具有 潜力的激光材料。

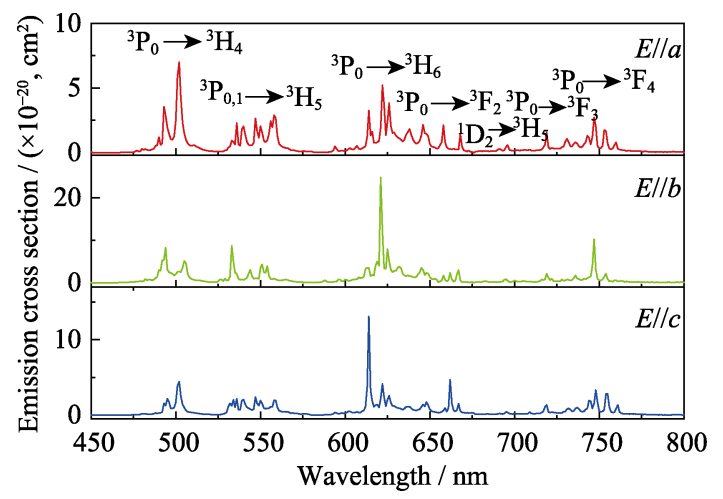

图 $3 \mathrm{Pr}$ : YAP 的偏振苂光光谱 ${ }^{[23]}$

Fig. 3 Polarized fluorescence spectra of Pr : YAP crystal ${ }^{[23]}$

表 1 Pr : YAP、Pr : SRA 和其他已实现 $\operatorname{Pr}^{3+}$ 激光输出晶体的光谱参数

Table 1 Lasing wavelength $\lambda_{\mathrm{em}}$, emission cross-section $\sigma_{\mathrm{em}}$ and $\sigma_{\mathrm{em}} \tau$ for the ${ }^{3} \mathrm{P}_{0} \rightarrow{ }^{3} \mathrm{H}_{6}$ transition of $\mathrm{Pr}^{3+}$ doped YAP and other crystals

\begin{tabular}{|c|c|c|c|c|c|c|c|c|c|c|}
\hline Transition & Parameters & LLF & YLF & GLF & $\mathrm{LaF}_{3}$ & BYF & LMA & ASL & YAP & SRA \\
\hline & Absorption & & & & & & & & & \\
\hline \multirow{3}{*}{${ }^{3} \mathrm{H}_{4} \rightarrow{ }^{3} \mathrm{P}_{2}$} & $\lambda_{\mathrm{abs}} / \mathrm{nm}$ & 444 & 444 & 444 & 442 & 445 & 444 & 444 & 449 & 445 \\
\hline & $\sigma_{\mathrm{abs}} /\left(\times 10^{-20}, \mathrm{~cm}^{2}\right)$ & 10.3 & 9.0 & 7.8 & 1.6 & 3.7 & 1.2 & 1.3 & 5.64 & 1.14 \\
\hline & $\mathrm{FWHM}_{\mathrm{abs}} / \mathrm{nm}$ & 1.70 & 1.80 & 1.9 & 5 & - & 7.1 & - & 5.6 & 9.59 \\
\hline & Emission & & & & & & & & & \\
\hline \multirow{4}{*}{${ }^{3} \mathrm{P}_{1} \rightarrow{ }^{3} \mathrm{H}_{5}$} & $\lambda_{\mathrm{em}} / \mathrm{nm}$ & 522 & 522 & 522 & 537 & 522 & 530 & 542 & 533 & 525 \\
\hline & $\sigma_{\mathrm{em}} /\left(\times 10^{-20}, \mathrm{~cm}^{2}\right)$ & 3 & 3 & 3 & 0.7 & 0.4 & 0.25 & 2 & 8.8 & 1.35 \\
\hline & $\mathrm{FWHM}_{\mathrm{em}} / \mathrm{nm}$ & $\sim 2$ & $\sim 2$ & 1 & $\sim 3$ & $\sim 4$ & 4 & - & 1.9 & 9.8 \\
\hline & $\sigma_{\mathrm{em}} \tau_{\mathrm{f}} /\left(\times 10^{-20}, \mathrm{~cm}^{2} \cdot \mu \mathrm{s}\right)$ & 113.7 & 107.1 & 130.8 & 35.7 & 17.9 & 8.6 & 76 & 168.6 & 41.5 \\
\hline \multirow{4}{*}{${ }^{3} \mathrm{P}_{0} \rightarrow{ }^{3} \mathrm{H}_{6}$} & $\lambda_{\mathrm{em}} / \mathrm{nm}$ & 607 & 607 & 607 & 610 & 607 & 625 & 620 & 621 & 622 \\
\hline & $\sigma_{\mathrm{em}} /\left(\times 10^{-20}, \mathrm{~cm}^{2}\right)$ & 12 & 14 & 13 & 2.9 & 24.7 & 3.7 & 2.9 & 25.01 & 3.52 \\
\hline & $\mathrm{FWHM}_{\mathrm{em}} / \mathrm{nm}$ & $\sim 3$ & $\sim 3$ & - & $\sim 6.9$ & 1.2 & 11 & - & 1.56 & 6.53 \\
\hline & $\sigma_{\mathrm{em}} \tau_{\mathrm{f}} /\left(\times 10^{-20}, \mathrm{~cm}^{2} \cdot \mu \mathrm{s}\right)$ & 454.8 & 499.8 & 566.8 & 147.9 & 1062.1 & 127.7 & 110.2 & 479.2 & 108.3 \\
\hline \multirow{4}{*}{${ }^{3} \mathrm{P}_{0} \rightarrow{ }^{3} \mathrm{~F}_{2}$} & $\lambda_{\mathrm{em}} / \mathrm{nm}$ & 640 & 640 & 640 & 635 & 639 & 647 & 643 & 662 & 644 \\
\hline & $\sigma_{\mathrm{em}} /\left(\times 10^{-20}, \mathrm{~cm}^{2}\right)$ & 21 & 22 & 23 & 1.2 & 12.1 & 2.3 & 8.5 & 4.71 & 10.37 \\
\hline & $\mathrm{FWHM}_{\mathrm{em}} / \mathrm{nm}$ & $\sim 0.7$ & $\sim 0.7$ & - & $\sim 1$ & 0.6 & 6.6 & - & 1.28 & 5.07 \\
\hline & $\sigma_{\mathrm{em}} \tau_{\mathrm{f}} /\left(\times 10^{-20}, \mathrm{~cm}^{2} \cdot \mu \mathrm{s}\right)$ & 795.9 & 785.4 & 1003 & 61.2 & 520.3 & 79.4 & 323 & 90.2 & 319.0 \\
\hline \multirow{6}{*}{${ }^{3} \mathrm{P}_{0} \rightarrow{ }^{3} \mathrm{~F}_{4}$} & $\lambda_{\mathrm{em}} / \mathrm{nm}$ & 720 & 720 & 720 & 720 & 721 & 728 & 725 & 747 & 725 \\
\hline & $\sigma_{\mathrm{em}} /\left(\times 10^{-20}, \mathrm{~cm}^{2}\right)$ & 7 & 9 & 16 & 6.6 & 7.3 & 3.3 & 11 & 10.37 & 5.75 \\
\hline & $\mathrm{FWHM}_{\mathrm{em}} / \mathrm{nm}$ & $\sim 1$ & $\sim 1$ & - & $\sim 3$ & 1.3 & 8.5 & - & 1.54 & 4.35 \\
\hline & $\sigma_{\mathrm{em}} \tau_{\mathrm{f}} /\left(\times 10^{-20}, \mathrm{~cm}^{2} \cdot \mu \mathrm{s}\right)$ & 265.3 & 321.3 & 697.6 & 336.6 & 309.6 & 113.9 & 418 & 198.7 & 176.9 \\
\hline & $\tau_{\mathrm{f}} / \mu \mathrm{s}$ & 37.90 & 35.70 & 43.6 & 51 & 43 & 34.5 & 38 & 19.16 & 30.76 \\
\hline & Ref. & {$[23]$} & [23] & {$[23]$} & {$[24]$} & {$[25-26]$} & {$[27]$} & {$[28]$} & \multicolumn{2}{|c|}{ This work } \\
\hline
\end{tabular}


$\operatorname{Pr}^{3+}$ 掺杂激光增益介质存在的一个问题是随 掺杂浓度增加而产生的交叉驰豫过程会导致上能 级 ${ }^{3} \mathrm{P}_{\mathrm{j}} /{ }^{1} \mathrm{I}_{6}$ 苂光寿命的降低(掺杂浓度不应超过 $1.5 \times$ $10^{20} \mathrm{~cm}^{-3[17]}$ )。另外, $\mathrm{Pr}^{3+}$ 的 $4 \mathrm{f}^{1} 5 \mathrm{~d}^{1}$ 能级位置相对于 $4 \mathrm{f}^{2}$ 态是较低的, 容易产生 ${ }^{3} \mathrm{P}_{2}$ 激发态吸收, 使 ${ }^{3} \mathrm{P}_{\mathrm{j}}$ 能级 的寿命缩短。同时, 激光下能级寿命为 $\mathrm{ms}$ 量级 ${ }^{[29-31]}$, 远远长于 $\mu \mathrm{s}$ 量级的上能级寿命, 存在严重的下能级 阻塞。

在基质材料的选择方面需要考虑两个主要的问 题。首先, 如果材料声子能量太高, ${ }^{3} \mathrm{P}_{\mathrm{j}} /{ }^{1} \mathrm{I}_{6}$ 能级容易 非辐射弛豫到 ${ }^{1} \mathrm{D}_{2}$ 能级。离子在基质中对应的带隙 不能低于四个声子的能量值 ${ }^{[33]}$ 。第二, $\operatorname{Pr}^{3+}$ 对应 $4 \mathrm{f}^{1} 5 \mathrm{~d}^{1}$ 态与 $4 \mathrm{f}^{2}$ 态的能级间距相比于其他离子是较低 的。基质的晶格场太强会引起泵浦光发生激发态吸 收。大的能级䢃裂会降低 $4 \mathrm{f}^{1} 5 \mathrm{~d}^{1}$ 能级的位置。之前 $\operatorname{Pr}^{3+}$ 掺杂材料主要使用氟化物晶体, 主要原因在于 氟化物晶体的声子能量低且晶格场较弱 ${ }^{[34]}$ 。Laroche 等 ${ }^{[35-36]}$ 研究表明 $\operatorname{Pr}^{3+}$ 最低的 $4 \mathrm{f}^{1} 5 \mathrm{~d}^{1}$ 能级位置为 $46000 \mathrm{~cm}^{-1}$, 即波长为 $220 \mathrm{~nm}$ 以下, 因此如果波长 大于 $440 \mathrm{~nm}$, 激发态吸收发生的概率就会大大降 低。以下将对这两个方面进行具体分析。

(1) $\operatorname{Pr}^{3+}$ 对应 ${ }^{3} \mathrm{P}_{0}$ 到 ${ }^{1} \mathrm{D}_{2}$ 能级间帯隙约为 $3500 \mathrm{~cm}^{-1}$,
如果基质材料声子能量太大，会引起两个能级间无 辐射跃迁, 从而降低 ${ }^{3} \mathrm{P}_{0}$ 能级的苂光量子效率。有研 究表明两能级带隙需要大于四个声子能量值才不会 发生无辐射弛豫损耗 ${ }^{[37]}$, 比如, 氟化物晶体容易实 现 $\operatorname{Pr}^{3+}$ 激光输出很大一部分原因在于声子能量低。 如图 5 所示, 氟化物材料的声子能量一般低于 $500 \mathrm{~cm}^{-1}$, 而氧化物材料中声子能量普遍大于 $500 \mathrm{~cm}^{-1}$ 。

对 $\mathrm{Pr}^{3+}$ 掺杂激光材料 ${ }^{3} \mathrm{P}_{0} \rightarrow{ }^{1} \mathrm{D}_{2}$ 无辐射跃迁速率 进行计算(公式 2):

$$
W_{\mathrm{NR}}=\beta_{\mathrm{el}} \exp [-\alpha(\Delta E-2 \hbar \omega)]
$$

其中, $W_{\mathrm{NR}}$ 代表无辐射跃迁速率, $\beta_{\mathrm{el}} 、 \alpha$ 代表掺杂离 子所在基质中的常数 ${ }^{[38]}, \Delta E$ 为 ${ }^{3} \mathrm{P}_{0}$ 到 ${ }^{1} \mathrm{D}_{2}$ 能级间带隙, $\hbar \omega$ 代表基质声子能量。

从计算结果(图 6)可以看出声子能量对 ${ }^{3} \mathrm{P}_{0} \rightarrow$ ${ }^{1} \mathrm{D}_{2}$ 能级间无辐射弛豫速率有较大影响。对于声子 能量较低的氟化物材料和 YAP、SRA 等氧化物材料, 无辐射跃迁速率较小。YAG、YSO 大声子能量基质 的无辐射跃迁速率较高(达到 $10^{5} \mathrm{~s}^{-1}$ 量级)。结果表 明 $\mathrm{Pr}^{3+}$ 在大声子能量氧化物中容易产生 ${ }^{3} \mathrm{P}_{0}$ 到 ${ }^{1} \mathrm{D}_{2}$ 能级的无辐射跃迁, 不易产生粒子数反转, 难以实 现激光输出, $\mathrm{Pr}^{3+}$ 掺杂基质需要满足低声子能量的 要求。

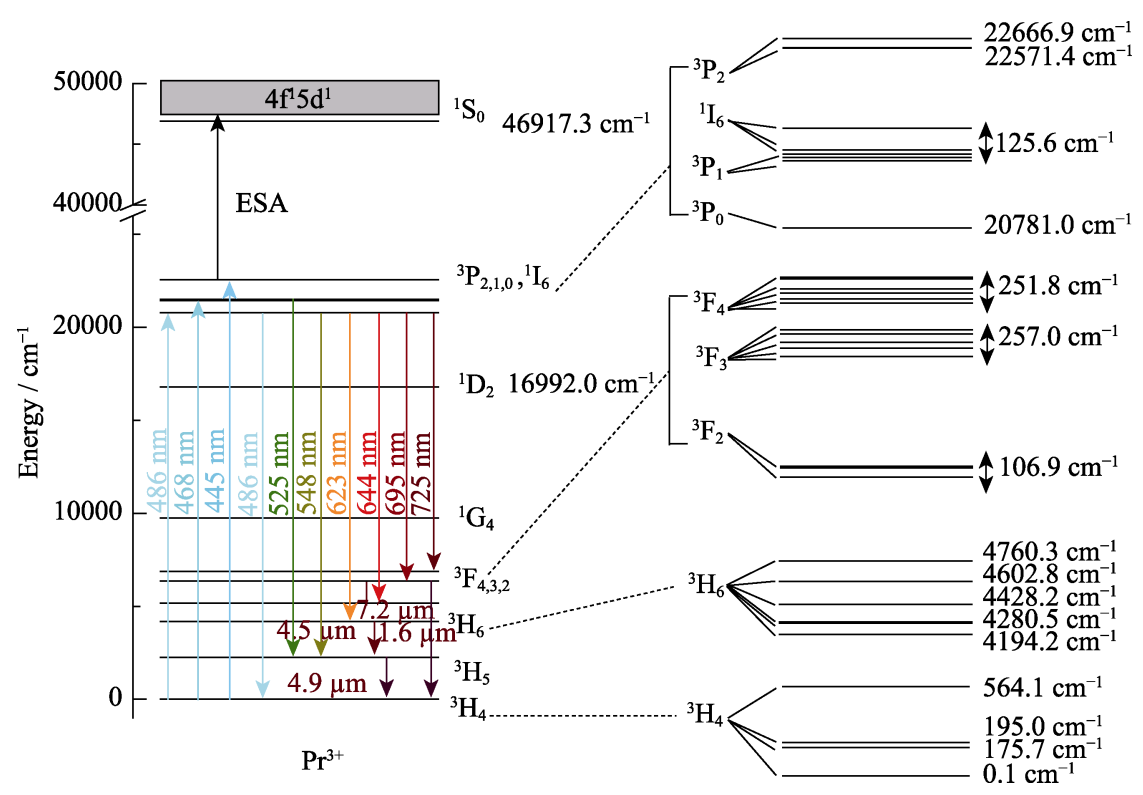

图 4 SRA 中 $\operatorname{Pr}^{3+}$ 的能级跃迁图 ${ }^{[32]}$

Fig. 4 Energy level diagram of Pr : SRA crystal ${ }^{[32]}$

(2)另一方面, $\operatorname{Pr}^{3+}$ 的 $4 \mathrm{f}^{1} 5 \mathrm{~d}^{1}$ 能级位置相对于 $4 \mathrm{f}^{2}$ 态是较低的, 容易产生 ${ }^{3} \mathrm{P}_{2}$ 激发态吸收。图 7 为 $\operatorname{Pr}^{3+}$ 掺杂基质中 ${ }^{1} \mathrm{~S}_{0}$ (或 $4 \mathrm{f}^{1} 5 \mathrm{~d}^{1}$ 最低能级位置)至 ${ }^{3} \mathrm{P}_{2}$ 带隙 分布图。室温 $\operatorname{Pr}^{3+}$ 掺杂材料 ${ }^{3} \mathrm{H}_{4} \rightarrow{ }^{3} \mathrm{P}_{2}$ 吸收峰位于 $445 \mathrm{~nm}$ $\left(22472 \mathrm{~cm}^{-1}\right.$ ) 附近(氟化物材料中 ${ }^{1} \mathrm{~S}_{0}$ 能级位置多位 于 $4 \mathrm{f}^{1} 5 \mathrm{~d}^{1}$ 之下, 最近研究表明, SRA、YAP 等多格位、
大离子间距氧化物晶体由于晶格场较弱, ${ }^{1} \mathrm{~S}_{0}$ 能级也 位于 $4 \mathrm{f}^{1} 5 \mathrm{~d}^{1}$ 态之下)。可以看出, 易实现 $\mathrm{Pr}^{3+}$ 掺杂激 光输出的激光材料, 如 YAP、SRA 氧化物晶体中 $\operatorname{Pr}^{3+}$ 的 $4 \mathrm{f}^{1} 5 \mathrm{~d}^{1}$ 态带隙与氟化物中比较接近, 均大于 $22472 \mathrm{~cm}^{-1}$, 而 YSO 和 YAG 中对应带隙远远小于 $22472 \mathrm{~cm}^{-1}$ 。在离子晶体中, $4 \mathrm{f}^{1} 5 \mathrm{~d}^{1}$ 组态主要处于 


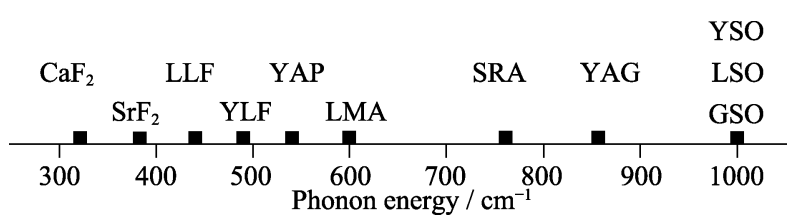

图 5 一些晶体材料的声子能量分布图

Fig. 5 Phonon energy distribution of some crystals

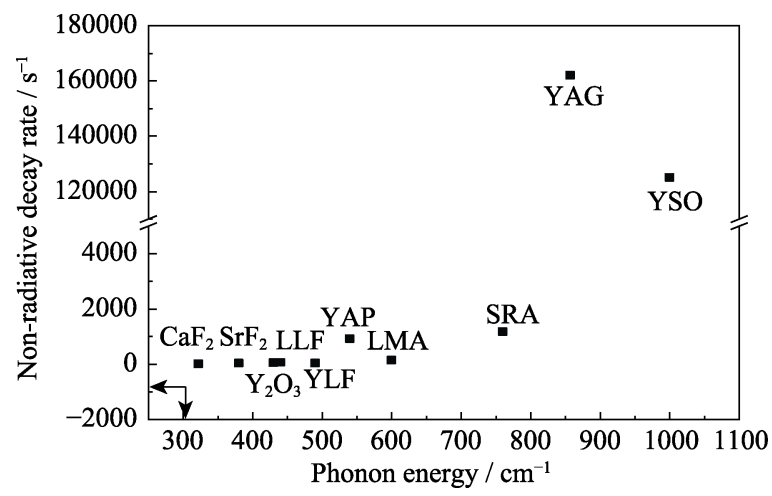

图 $6 \mathrm{Pr}^{3+}$ 掺杂激光材料 ${ }^{3} \mathrm{P}_{0} \rightarrow{ }^{1} \mathrm{D}_{2}$ 无辐射弛豫速率与声子能 量的关系图

Fig. 6 Non-radiative decay rate $v s$ phonon energy of some $\operatorname{Pr}^{3+}$ doped crystals

紫外区，随着晶体场增强，电子云膨胀效应使其下 降, 从而降低 $4 \mathrm{f}-5 \mathrm{~d}$ 带隙。能级䢃裂程度与晶场强度 成正比，而晶格场的强弱除了与电负性有关 $\left(\mathrm{F}^{-}>\mathrm{O}^{2-}>\mathrm{Cl}^{-}>\mathrm{Br}^{-}>\mathrm{I}^{-}\right.$, 电负性越大, 晶场强度越弱 $)$, 还与内部离子间距有一定关联 ${ }^{[20]}$ 。其中, YAP 中 $\mathrm{Y}-\mathrm{O}$ 间距为 $0.262 \mathrm{~nm}$, 相比于 $Y A G$ 中 $Y-O$ 间距 $0.245 \mathrm{~nm}$ 要大得多。 $\mathrm{Pr}^{3+}$ 在 YAP 对应的能级䢃裂较小, 带隙 高达 $24099 \mathrm{~cm}^{-1[39]}$ 。然而 $\mathrm{Pr}^{3+}$ 在 YAG 中 ${ }^{1} \mathrm{~S}_{0}$ 能级相 对 ${ }^{3} \mathrm{H}_{4}$ 处于 $33000 \mathrm{~cm}^{-1},{ }^{3} \mathrm{P}_{2}$ 能级为 $22124 \mathrm{~cm}^{-1}$, 带隙 小于 $11000 \mathrm{~cm}^{-1[40]}$, 很容易发生 ${ }^{3} \mathrm{P}_{2}$ 能级的激发态 吸收。大量报道也证明 $\mathrm{Pr}^{3+}$ 在 YAG、YSO 中难以实 现激光输出。以上分析可以看出, $\operatorname{Pr}^{3+}$ 掺杂激光材料 需满足 $4 \mathrm{f}-5 \mathrm{~d}$ 带隙至少大于 $22472 \mathrm{~cm}^{-1}$, 即 $4 \mathrm{f}^{1} 5 \mathrm{~d}^{1}$ 态 位置至少是 ${ }^{3} \mathrm{P}_{2}$ 能级位置的两倍(如式 3 所示), 才不 容易发生激发态吸收。

$$
E_{5 \mathrm{~d}}>2 \times E_{0}
$$

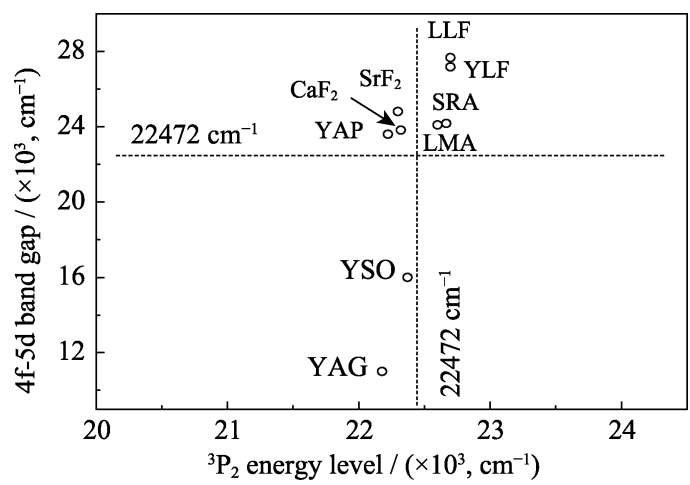

图 $7 \mathrm{Pr}^{3+}$ 掺杂激光材料 $4 \mathrm{f}-5 \mathrm{~d}$ 带隙与 ${ }^{3} \mathrm{P}_{2}$ 能级位置关系图

Fig. 7 ff-5d band gap $v s^{3} \mathrm{P}_{2}$ energy level of some $\mathrm{Pr}^{3+}$ doped crystals
其中, $E_{5 \mathrm{~d}}$ 为 $4 \mathrm{f}^{1} 5 \mathrm{~d}^{1}$ 态能量, $E_{0}$ 为 ${ }^{3} \mathrm{P}_{2}$ 能级处能量。

无辐射跃迁几率和激发态吸收都会影响产生激 光的上能级寿命。图 8 为典型的激光材料激光上能 级寿命与基质声子能量的关系图。氟化物的上能级 寿命普遍高于氧化物。这是由于氟化物声子能量较 低, 无辐射跃迁速率较低, 因此其上能级寿命较长。

此外, 相较于氟化物, 氧化物的机械性能和热 学性能更好, 在大功率激光输出上可以更有效地抑 制热透镜等现象的发生，属于性能非常优异的潜在 激光材料, 具有很高的研究价值

\section{2 掺 $\operatorname{Pr}^{3+}$ 激光晶体的激光特性}

到目前为止, $\operatorname{Pr}^{3+}$ 具有相对优异的可见激光输 出特性。1963 年, 在闪光灯泵浦下, $\operatorname{Pr}^{3+}$ 掺杂 $\mathrm{LaF}_{3}$ 首次实现 $599 \mathrm{~nm}$ 激光输出 ${ }^{[41]}$ 。起初, 在蓝光波段激 发 $\mathrm{Pr}^{3+}$ 实现激光输出主要使用染料激光器泵浦 $\mathrm{PrCl}_{3}$ 或 $\mathrm{PrBr}_{3}$ 。后来，德国 Sandrock 等 ${ }^{[42]}$ 首次使用 $\mathrm{Ar}^{+}$ 激光器洜浦 Pr: YLF 成功实现绿光、橙光、红光以 及深红光的激光运转。其中, $522 \mathrm{~nm}$ 输出功率为 $144 \mathrm{~mW}, 639 \mathrm{~nm}$ 输出功率为 $266 \mathrm{~mW}$ 。 1996 年, 其 团队使用 $\mathrm{Ti}: \mathrm{Al}_{2} \mathrm{O}_{3}$ 激光器激发 $\mathrm{Pr}, \mathrm{Yb}: \mathrm{LiYF}_{4}$ 晶体, 分别获得 $75 \mathrm{~mW}$ 的 $639.5 \mathrm{~nm}$ 激光和 $19 \mathrm{~mW}$ 的 $720 \mathrm{~nm}$ 激光输出 ${ }^{[43]}$ 。2 21 世纪后, 随着蓝光蜃浦源激光器技 术的进步和发展, $\mathrm{Pr}^{3+}$ 可见光激光获得飞速发展。表 2 为近年来 $\operatorname{Pr}^{3+}$ 掺杂激光晶体在可见波段的激光输出。

在掺杂 $\operatorname{Pr}^{3+}$ 实现可见波段激光输出的晶体材料 中, 氟化物 YLF 在蓝、绿、橙、红和深红波段均已 实现激光输出。从表 2 可以看出, 使用 $2 \omega-$ OPSL 原 浦 Pr: YLF, 实现 $523 \mathrm{~nm}$ 处约 $4.2 \mathrm{~W}$ 的绿光激光运 转, 斜率效率为 $45 \%$ 。此外使用蓝光 $\mathrm{LD}$ 泵浦相同 材料, 在 $640 \mathrm{~nm}$ 处斜率效率为 $50 \%$, 输出功率高达 $4.8 \mathrm{~W}$, 这是目前 $\operatorname{Pr}^{3+}$ 掺杂激光晶体的最高输出功 率。相比国际上 $\mathrm{Pr}^{3+}$ 激光的发展, 国内发展起步较 晚, 厦门大学 $\mathrm{Xu}$ 等 ${ }^{[47]}$ 使用 InGaN LD 泵浦 Pr : YLF

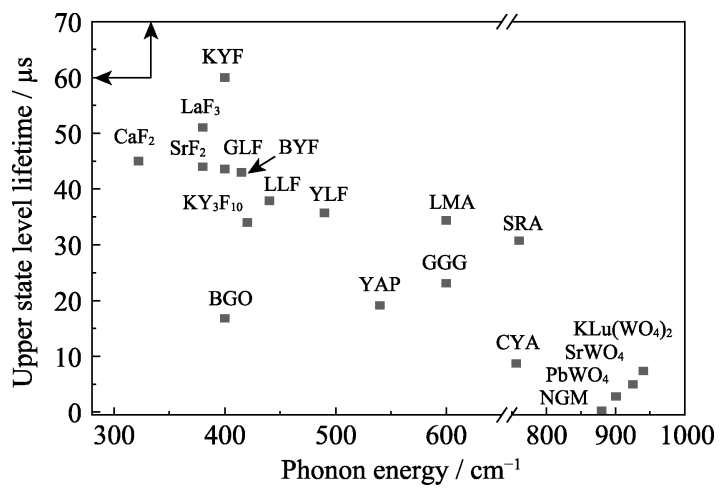

图 $8 \mathrm{Pr}^{3+}$ 掺杂激光材料上能级寿命与声子能量的关系图 Fig. 8 Upper state level lifetime vs phonon energy of some $\operatorname{Pr}^{3+}$ doped crystals 
表 $2 \mathrm{Pr}^{3+}$ 掺杂激光晶体在可见波段的连续激光输出

Table 2 Visible laser output of $\operatorname{Pr}^{3+}$ doped crystals

\begin{tabular}{|c|c|c|c|c|c|c|c|c|}
\hline Host & $\lambda_{\mathrm{em}} / \mathrm{nm}$ & Polarization & Laser transition & $\eta_{\text {slope }} / \%$ & $P_{\text {out }} / \mathrm{mW}$ & $P_{\text {thr }} / \mathrm{mW}$ & Pump source & Year \\
\hline \multirow{7}{*}{ YLF } & 491 & $\sigma$ & ${ }^{3} \mathrm{P}_{\mathrm{J}} \rightarrow{ }^{3} \mathrm{H}_{4}$ & 6 & 70 & 285 & $2 \omega-\mathrm{OPSL}$ & $2014^{[44]}$ \\
\hline & 523 & $\pi$ & ${ }^{3} \mathrm{P}_{\mathrm{J}} \rightarrow{ }^{3} \mathrm{H}_{5}$ & 45 & $\sim 4200$ & $>500$ & $2 \times 2 \omega-\mathrm{OPSL}$ & $2016^{[17]}$ \\
\hline & 546 & $\pi$ & ${ }^{3} \mathrm{P}_{\mathrm{J}} \rightarrow{ }^{3} \mathrm{H}_{5}$ & 60 & 2000 & 120 & $2 \omega-\mathrm{OPSL}$ & $2014^{[45]}$ \\
\hline & 605 & $\sigma$ & ${ }^{3} \mathrm{P}_{\mathrm{J}} \rightarrow{ }^{3} \mathrm{H}_{6}$ & 25 & 2100 & $\sim 1500$ & Blue-LD & $2017^{[46]}$ \\
\hline & 640 & $\sigma$ & ${ }^{3} \mathrm{P}_{\mathrm{J}} \rightarrow{ }^{3} \mathrm{~F}_{2}$ & 50 & 4800 & $\sim 500$ & Blue-LD & $2017^{[46]}$ \\
\hline & 698 & $\sigma$ & ${ }^{3} \mathrm{P}_{\mathrm{J}} \rightarrow{ }^{3} \mathrm{~F}_{3}$ & 36 & 1300 & 78 & InGaN-LD & $2016^{[47]}$ \\
\hline & 721 & $\pi$ & ${ }^{3} \mathrm{P}_{\mathrm{J}} \rightarrow{ }^{3} \mathrm{~F}_{4}$ & 53 & 1000 & 16 & $2 \omega-\mathrm{OPSL}$ & $2014^{[47]}$ \\
\hline \multirow[t]{4}{*}{ LLF } & 523 & $E / / c$ & ${ }^{3} \mathrm{P}_{0} \rightarrow{ }^{3} \mathrm{H}_{5}$ & 56 & 52.7 & 10 & 2-OPSL & $2007^{[48]}$ \\
\hline & 607 & $E / / c$ & ${ }^{3} \mathrm{P}_{0} \rightarrow{ }^{3} \mathrm{H}_{6}$ & 31 & 34.5 & 26 & 2-OPSL & $2007^{[48]}$ \\
\hline & 640 & $E / / c$ & ${ }^{3} \mathrm{P}_{0} \rightarrow{ }^{3} \mathrm{~F}_{2}$ & 56 & 52.7 & 39 & 2-OPSL & $2007^{[48]}$ \\
\hline & 722 & $E / / c$ & ${ }^{3} \mathrm{P}_{0} \rightarrow{ }^{3} \mathrm{~F}_{4}$ & 46 & 50 & 31 & 2-OPSL & $2007^{[48]}$ \\
\hline \multirow[t]{3}{*}{ BYF } & 495 & $E / / X$ & ${ }^{3} \mathrm{P}_{\mathrm{J}} \rightarrow{ }^{3} \mathrm{H}_{4}$ & 27 & 201 & 163 & $2 \omega-\mathrm{OPSL}$ & $2014^{[44]}$ \\
\hline & 607 & $E / / Y$ & ${ }^{3} \mathrm{P}_{\mathrm{J}} \rightarrow{ }^{3} \mathrm{H}_{6}$ & 12.6 & 99 & 264 & Blue-LD & $2014^{[49]}$ \\
\hline & 639 & $E / / Y$ & ${ }^{3} \mathrm{P}_{\mathrm{J}} \rightarrow{ }^{3} \mathrm{~F}_{2}$ & 6.4 & 60 & 146 & Blue-LD & $2014^{[49]}$ \\
\hline \multirow[t]{3}{*}{ KYF } & 554 & - & ${ }^{3} \mathrm{P}_{\mathrm{J}} \rightarrow{ }^{3} \mathrm{H}_{5}$ & 27 & 121 & 166 & InGaN-LD & $2013^{[50]}$ \\
\hline & 610 & - & ${ }^{3} \mathrm{P}_{\mathrm{J}} \rightarrow{ }^{3} \mathrm{H}_{6}$ & 18 & 97 & 162 & InGaN-LD & $2013^{[50]}$ \\
\hline & 645 & - & ${ }^{3} \mathrm{P}_{\mathrm{J}} \rightarrow{ }^{3} \mathrm{~F}_{2}$ & 38 & 268 & 30 & InGaN-LD & $2013^{[50]}$ \\
\hline \multirow[t]{6}{*}{ YGF } & 523 & $E / / a$ & ${ }^{3} \mathrm{P}_{\mathrm{J}} \rightarrow{ }^{3} \mathrm{H}_{5}$ & 11 & 63 & 148 & InGaN-LD & $2015^{[21]}$ \\
\hline & 538 & $E / / b$ & ${ }^{3} \mathrm{P}_{\mathrm{J}} \rightarrow{ }^{3} \mathrm{H}_{5}$ & 24 & 140 & 135 & InGaN-LD & $2015^{[21]}$ \\
\hline & 604 & $E / / a$ & ${ }^{3} \mathrm{P}_{\mathrm{J}} \rightarrow{ }^{3} \mathrm{H}_{6}$ & 13 & 105 & 72 & InGaN-LD & $2015^{[21]}$ \\
\hline & 638 & $E / / a$ & ${ }^{3} \mathrm{P}_{\mathrm{J}} \rightarrow{ }^{3} \mathrm{~F}_{2}$ & 16 & 128 & 188 & InGaN-LD & $2015^{[21]}$ \\
\hline & 700 & $E / / a$ & ${ }^{3} \mathrm{P}_{\mathrm{J}} \rightarrow{ }^{3} \mathrm{~F}_{4}$ & 18 & 78 & 47 & InGaN-LD & $2015^{[21]}$ \\
\hline & 724 & $E / / b$ & ${ }^{3} \mathrm{P}_{\mathrm{J}} \rightarrow{ }^{3} \mathrm{~F}_{4}$ & 20 & 117 & 48 & InGaN-LD & $2015^{[21]}$ \\
\hline $\mathrm{CaF}_{2}$ & 642 & - & ${ }^{3} \mathrm{P}_{\mathrm{J}} \rightarrow{ }^{3} \mathrm{~F}_{2}$ & 7.5 & 22 & 305 & InGaN-LD & $2017^{[51]}$ \\
\hline \multirow[t]{4}{*}{$\mathrm{LaF}_{3}$} & 537 & $\pi$ & ${ }^{3} \mathrm{P}_{\mathrm{J}} \rightarrow{ }^{3} \mathrm{H}_{5}$ & 16 & 15 & 159 & InGaN-LD & $2012^{[24]}$ \\
\hline & 612 & $\pi$ & ${ }^{3} \mathrm{P}_{\mathrm{J}} \rightarrow{ }^{3} \mathrm{H}_{6}$ & 15 & 20 & 98 & InGaN-LD & $2012^{[24]}$ \\
\hline & 635 & $c$ & ${ }^{3} \mathrm{P}_{\mathrm{J}} \rightarrow{ }^{3} \mathrm{~F}_{2}$ & 16 & 23 & 95 & InGaN-LD & $2012^{[24]}$ \\
\hline & 720 & $\pi$ & ${ }^{3} \mathrm{P}_{\mathrm{J}} \rightarrow{ }^{3} \mathrm{~F}_{4}$ & 37 & 80 & 10 & InGaN-LD & $2012^{[24]}$ \\
\hline \multirow{3}{*}{ ASL } & 620 & $\pi$ & ${ }^{3} \mathrm{P}_{\mathrm{J}} \rightarrow{ }^{3} \mathrm{H}_{6}$ & 11 & 50 & $\sim 510$ & $2 \omega-\mathrm{OPSL}$ & $2018^{[52]}$ \\
\hline & 643 & $\pi$ & ${ }^{3} \mathrm{P}_{\mathrm{J}} \rightarrow{ }^{3} \mathrm{~F}_{2}$ & 27 & 160 & $\sim 200$ & $2 \omega-\mathrm{OPSL}$ & $2018^{[52]}$ \\
\hline & 725 & $\pi$ & ${ }^{3} \mathrm{P}_{\mathrm{J}} \rightarrow{ }^{3} \mathrm{~F}_{4}$ & 37 & 318 & $\sim 280$ & $2 \omega-\mathrm{OPSL}$ & $2018^{[52]}$ \\
\hline \multirow[t]{3}{*}{ LMA } & 620 & $\sigma$ & ${ }^{3} \mathrm{P}_{\mathrm{J}} \rightarrow{ }^{3} \mathrm{H}_{6}$ & 2 & 2.9 & $\sim 100$ & $2 \omega$-OPSL & $2012^{[27]}$ \\
\hline & 648 & $\sigma$ & ${ }^{3} \mathrm{P}_{\mathrm{J}} \rightarrow{ }^{3} \mathrm{~F}_{2}$ & 4 & 10.1 & $\sim 90$ & $2 \omega-\mathrm{OPSL}$ & $2012^{[27]}$ \\
\hline & 729 & $\sigma$ & ${ }^{3} \mathrm{P}_{\mathrm{J}} \rightarrow{ }^{3} \mathrm{~F}_{4}$ & 12 & 63.7 & $\sim 25$ & $2 \omega-\mathrm{OPSL}$ & $2012^{[27]}$ \\
\hline \multirow{3}{*}{ YAP } & 547 & $E / / c$ & ${ }^{3} \mathrm{P}_{\mathrm{J}} \rightarrow{ }^{3} \mathrm{H}_{5}$ & 6.1 & 37 & 320 & InGaN-LD & $2013^{[53]}$ \\
\hline & 662 & $E / / c$ & ${ }^{3} \mathrm{P}_{\mathrm{J}} \rightarrow{ }^{3} \mathrm{~F}_{2}$ & 9 & 27.4 & 680 & GaN-LD & $2011^{[54]}$ \\
\hline & 747 & $E / / b$ & ${ }^{3} \mathrm{P}_{\mathrm{J}} \rightarrow{ }^{3} \mathrm{~F}_{4}$ & 45 & 490 & 300 & $2 \times \operatorname{InGaN}-\mathrm{LD}$ & $2014^{[55]}$ \\
\hline \multirow{4}{*}{ SRA } & 525 & - & ${ }^{3} \mathrm{P}_{\mathrm{J}} \rightarrow{ }^{3} \mathrm{H}_{5}$ & - & 36 & $\sim 1000$ & $2 \omega-\mathrm{OPSL}$ & $2013^{[56]}$ \\
\hline & 623 & $\sigma$ & ${ }^{3} \mathrm{P}_{\mathrm{J}} \rightarrow{ }^{3} \mathrm{H}_{6}$ & 11 & 114 & $\sim 200$ & $4 \times$ InGaN-LD & $2014^{[15]}$ \\
\hline & 644 & - & ${ }^{3} \mathrm{P}_{\mathrm{J}} \rightarrow{ }^{3} \mathrm{~F}_{2}$ & 37 & 1065 & $\sim 500$ & $2 \omega-\mathrm{OPSL}$ & $2013^{[56]}$ \\
\hline & 724 & $\sigma$ & ${ }^{3} \mathrm{P}_{\mathrm{J}} \rightarrow{ }^{3} \mathrm{~F}_{4}$ & 50 & 564 & 15.5 & $4 \times$ InGaN-LD & $2014^{[15]}$ \\
\hline
\end{tabular}

晶体, 实现了 523、607、639、698、721 nm 连续激 光输出, 输出功率均达到瓦级。其中, $639 \mathrm{~nm}$ 处功率 高达 $2.3 \mathrm{~W}$, 斜率效率为 $57 \%$ 。相对于功率较高的氟 化物晶体, 掺 $\mathrm{Pr}^{3+}$ 氧化物的研究较少。目前实现激
光输出的氧化物激光晶体主要为 YAP、SRA 和 $\mathrm{Sr}_{0.7} \mathrm{La}_{0.3} \mathrm{Mg}_{0.3} \mathrm{Al}_{11.7} \mathrm{O}_{19}$ (ASL)。2013 年, Reichert 等 ${ }^{[57]}$ 使用 $2 \omega$-OPSL 原浦 Pr: SRA 晶体, 实现 $644 \mathrm{~nm}$ 处 功率为 $1065 \mathrm{~mW}$ 的激光输出, 这是目前 $\operatorname{Pr}^{3+}$ 掺杂氧 
化物晶体的最高输出功率。最近, Sattayaporn 等 ${ }^{[52]}$ 在 Pr: ASL 晶体中获得 620、643、725 nm 连续激 光输出, 其中 $725 \mathrm{~nm}$ 处最高输出功率为 $318 \mathrm{~mW}$ 。 值得注意的是, 上述 $\operatorname{Pr}^{3+}$ 掺杂的氧化物都提供 12 个 格位, 这就使得 $\mathrm{Pr}^{3+}$ 处于相对弱的晶格场, 可以有 效地避免 $4 \mathrm{f}^{1} 5 \mathrm{~d}^{1}$ 䢃裂引起的能级位置的降低, 从而 降低激发态吸收的概率。与此相反的是, 氧化物材 料比如 YAG 或者倍半氧化物并不能提供多的格位 数, 且 $\mathrm{Pr}^{3+}$ 与配位 $\mathrm{O}^{2-}$ 间距较小, 晶场较强, 能级䢃 裂导致的激发态吸收较强, 有研究显示其不适于 $\mathrm{Pr}^{3+}$ 掺杂实现激光输出 ${ }^{[58]}$ 。

从上述讨论可以发现, 六十年前首次报道 $\operatorname{Pr}^{3+}$ 掺杂激光晶体激光输出后, 后续没有引起 $\operatorname{Pr}^{3+}$ 研究 的爆发式增长, 其主要原因是晶体生长技术还不能 够获得高质量的激光晶体, 并且缺乏高效的泵浦 源。然而, 随着科技的发展, 晶体生长已经日趋成熟, 晶体品质相比之前有了明显的改善。随着蓝光 InGaN 激光泵浦源以及倍频光泵浦半导体激光泵浦 源的出现, 多种类、大功率毬浦源使得 $\operatorname{Pr}^{3+}$ 掺杂激 光晶体实现高功率激光输出指日可待。加快激光新 波段研发进程、获得自主知识产权的高效率紧凑型 新波段激光器件以赶超国外激光水平具有非常深远 的意义 ${ }^{[59]}$ 。

\section{2 掺 $\mathrm{Dy}^{3+}$ 激光晶体}

\section{1 掺 $\mathrm{Dy}^{3+}$ 激光晶体的光谱特性}

在稀土离子中, $\operatorname{Pr}^{3+}$ 由于在可见区域拥有大量 的跃迁而成为应用较广泛的稀土离子, 然而 $\mathrm{Pr}^{3+}$ 在 黄光波段没有对应的能级跃迁。黄光激光在钠信标 激光器、科研以及遥感等领域拥有很高的应用价值。 $\mathrm{Dy}^{3+}$ 包含七个 $4 \mathrm{f}-4 \mathrm{f}$ 能级跃迁, 其中强度最高的 ${ }^{4} \mathrm{~F}_{9 / 2} \rightarrow{ }^{6} \mathrm{H}_{13 / 2}$ 跃迁恰好处于黄光发射波段。因此, 在 黄光波段激光研究中, 取得了很多有意义的进展。 $\mathrm{Dy}^{3+}$ 所有可见波段跃迁都需自旋反转, 因此 425 、 $450 、 475 \mathrm{~nm}$ 处基态吸收截面较小, 约为 $10^{-21} \mathrm{~cm}^{2}$ 。 由于 $\mathrm{Dy}^{3+}$ 容易产生交叉驰豫, 其最大的掺杂浓度不 应超过 $10^{21} \mathrm{~cm}^{-3[17]}$ 。因为吸收截面较小, 激光输出 需要的晶体样品长度往往达到厘米尺度以保证足够 的能量吸收。自旋禁戒使得发射与吸收截面处于相 同量级。 $\mathrm{Dy}^{3+}$ 在 $574 \mathrm{~nm}$ 附近黄光最大的发射截面约 为 $10^{-21} \mathrm{~cm}^{2}$, 其他稀土离子 (除 $\mathrm{Tb}^{3+}$ 外) 在这个波段 没有相应的激光能级跃迁。 ${ }^{4} \mathrm{~F}_{9 / 2}$ 上能级苂光寿命为 $1 \mathrm{~ms}$ 量级。

$\mathrm{Dy}^{3+}$ 激光输出面临的一个问题是, 黄光跃迁下 能级 ${ }^{6} \mathrm{H}_{13 / 2}$ 的寿命较长, 易产生粒子数阻塞。高声子
能量基质材料更有利于下能级 ${ }^{6} \mathrm{H}_{9 / 2} 、{ }^{6} \mathrm{H}_{11 / 2} 、{ }^{6} \mathrm{H}_{13 / 2}$ 产生弛豫。另一个去粒子数堆积的方法是共掺其他 激活离子, 使 ${ }^{6} \mathrm{H}_{13 / 2}$ 堆积的粒子数通过与共掺离子 能量传递回到 ${ }^{6} \mathrm{H}_{15 / 2}$ 基态。这种方法要避免 ${ }^{4} \mathrm{~F}_{9 / 2}$ 上 能级发生交叉驰豫, 产生苂光猝灭。 $\mathrm{Dy}^{3+}$ 的 $4 \mathrm{f}^{8} 5 \mathrm{~d}^{1}$ 低能级位置较高, 不容易发生激发态吸收, 因此氧 化物晶体适宜作为 $\mathrm{Dy}^{3+}$ 掺杂的基质材料。

图 9 为室温下 Dy：YAP 晶体偏振吸收谱。因为 晶格场离子之间的相互影响, $\mathrm{Dy}^{3+}$ 的一些吸收带出 现重叠现象 ${ }^{[60]}$ 。在波长 $450 \mathrm{~nm}$ 处, $a 、 b$ 和 $c$ 偏振对 应的吸收截面分别为 $0.743 \times 10^{-21} 、 0.690 \times 10^{-21}$ 和 $0.870 \times 10^{-21} \mathrm{~cm}^{2}$, 半高宽 FWHM 分别为 $10.7 、 11.3$ 和 $11.1 \mathrm{~nm}$ 。结果表明 Dy：YAP 晶体非常适合使用 InGaN 二极管洜浦。

Dy : YAP 晶体在偏振荧光光谱如图 10 所示。 发射峰分别位于 574、664 和 $754 \mathrm{~nm}$ 。在 $574 \mathrm{~nm}$ 发 射强度最大, $a 、 b$ 和 $c$ 三个偏振方向的发射截面分 别为 $0.298 \times 10^{-20} 、 0.450 \times 10^{-20}$ 和 $0.452 \times 10^{-20} \mathrm{~cm}^{2}$, 半 高宽 FWHM 分别为 $8.2 、 8.3$ 和 $8.1 \mathrm{~nm}$ 。Dy：YAP 发射截面与 $\mathrm{Dy}: \mathrm{GdVO}_{4}{ }^{[61]}$ 、 $\mathrm{Dy}: \mathrm{ZnWO}_{4}{ }^{[62]}$ 和 Dy: $\mathrm{YAG}^{[63]}$ 相近, 都处于 $10^{-20} \mathrm{~cm}^{2}$ 数量级。大的发 射截面和半峰宽表明 Dy：YAP 晶体在黄光激光输 出上有很大的潜力。

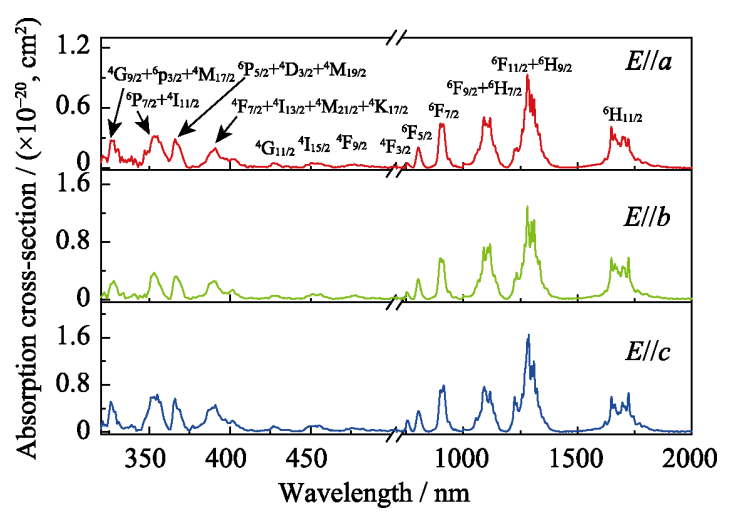

图 9 Dy : YAP 晶体的偏振吸收谱 ${ }^{[60]}$

Fig. 9 Polarized absorption spectra of Dy : YAP crystal ${ }^{[60]}$

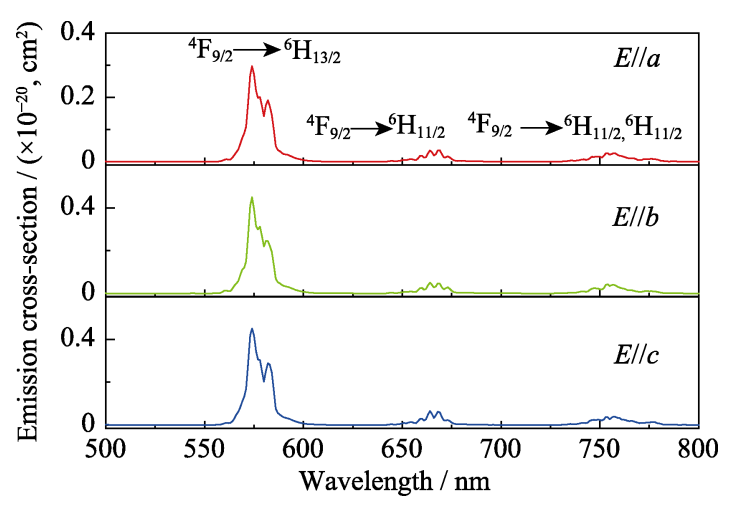

图 10 Dy : YAP 晶体在 $450 \mathrm{~nm}$ 激发下的偏振苂光光谱 ${ }^{[60]}$ Fig. 10 Polarized fluorescence spectra of Dy : YAP crystal ${ }^{[60]}$ 
表 3 给出了 $\mathrm{Dy}^{3+}$ 掺杂 $\mathrm{YAP}$ 晶体和其他基质的光 谱参数。Dy：YAP 黄光苂光分支比相比于其他材料 要大的多, 非常有利于实现黄光激光输出。 Dy : YAP 的发射截面与 Dy : KYF 和 Dy : LSO 比较 接近, 对应上能级 ${ }^{4} \mathrm{~F}_{9 / 2}$ 的苂光寿命相对较短, 但是 超过 $\mathrm{Dy}: \mathrm{LiGd}_{4}\left(\mathrm{MoO}_{4}\right)_{7}$ 。这在某种程度上与 $\mathrm{Dy}^{3+}$ 的掺杂浓度偏高有关, 荧光寿命的增大可以通过优 化 $\mathrm{Dy}^{3+}$ 掺杂浓度来进行调控。

\section{2 掺 $\mathrm{Dy}^{3+}$ 激光晶体的激光特性}

1996 年, $\mathrm{Dy}: \mathrm{LiNbO}_{3}$ 激光在低温下实现首次激 光输出 ${ }^{[69]}$ 。后来, $\mathrm{Dy}^{3+}$ 掺杂光纤激光器在黄光与蓝 光成功实现了激光输出 ${ }^{[70]}$ 。表 4 列出了近年来 $\mathrm{Dy}^{3+}$ 掺杂激光晶体在可见波段的激光输出。氧化物材料 中激光输出功率相对较大, 2012 年, 首个 InGaN LD 泵浦 Dy: YAG 晶体实现黄光激光运转, 输出功率 达到 $150 \mathrm{~mW}^{[64]}$ 。最近, $\mathrm{Xia}$ 等 ${ }^{[71]}$ 在 $\mathrm{Dy}^{3+}$ 掺杂 $\mathrm{ZnWO}_{4}$ 基质中实现 $575 \mathrm{~nm}$ 处连续激光运转, 输出功率为 $110 \mathrm{~mW}$, 斜率效率为 $13 \%$ 。然而, 掺 $\mathrm{Dy}^{3+}$ 氟化物晶 体 LLF 中 $578 \mathrm{~nm}$ 处黄光自脉冲激光的输出功率为 $17 \mathrm{~mW}$, 斜率效率仅为 $4 \%{ }^{[17]}$ 。这是因为随着㬌浦功 率的增大, 低热导率和低机械强度的氟化物出现热 透镜效应, 激光运转稳定性变差, 能量损耗变大。有 报道显示, 共掺 $\mathrm{Tb}^{3+}$ 或者 $\mathrm{Eu}^{3+}$ 可以明显缩短下能级 寿命, 提高激光运转效率。到目前为止, 共掺后最高
的输出功率是 $55 \mathrm{~mW}$, 斜率效率为 $13 \%{ }^{[72]}$ 。更高的 功率很可能通过优化 $\mathrm{Dy}^{3+}$ 与共掺离子配比来实现。

\section{3 掺 $\mathbf{T b}^{\mathbf{3}}$ 激光晶体}

\section{1 掺 $\mathbf{T b}^{\mathbf{3}}$ 激光晶体的光谱特性}

$\mathrm{Tb}^{3+}$ 是稀土离子中除 $\mathrm{Dy}^{3+}$ 以外仅有的另一个存 在黄光能级跃迁的离子。在 $\mathrm{Tb}^{3+}$ 的能级图中(如图 1), ${ }^{5} \mathrm{D}_{4}$ 和 ${ }^{5} \mathrm{D}_{3}$ 能级都被认为属于激光上能级。两个能级 间带隙为 $5500 \mathrm{~cm}^{-1}$, 因此基质声子能量很难使其产 生无辐射弛豫。比如 ${ }^{5} \mathrm{D}_{4}$ 能级不会受到交叉驰豫的 影响, 即使在高掺杂浓度下其能级苂光寿命也可达 到 $1 \mathrm{~ms}$ 。 ${ }^{5} \mathrm{D}_{4}$ 能级可使用 $484 \mathrm{~nm}$ 波长百浦, 但是 ${ }^{7} \mathrm{~F}_{6} \rightarrow{ }^{5} \mathrm{D}_{4}$ 属于禁戒跃迁, 因此吸收截面值仅为 $10^{-22} \mathrm{~cm}^{2}$ 。对于 $\mathrm{Tb}^{3+}$ 来讲, 低的吸收截面可以通过 高的掺杂浓度来弥补, 且不会引起 ${ }^{5} \mathrm{D}_{4}$ 能级浓度猝 灭, 其掺杂浓度可以高达 $10^{21} \mathrm{~cm}^{-3[17]}$, 吸收系数可 以达到 $1 \mathrm{~cm}^{-1}$ 。高的掺杂浓度以及长的上能级寿命 使得 $\mathrm{Tb}^{3+}$ 在调 $\mathrm{Q}$ 脉冲激光方面具有很高的研究价 值。 $\mathrm{Tb}^{3+}$ 类似于 $\mathrm{Pr}^{3+}$ 和 $\mathrm{Ce}^{3+}$, 其激发态吸收可以达到 $5 \mathrm{~d}$ 能级。事实上, 这些 $5 \mathrm{~d}$ 能级在氟化物中处于 $250 \mathrm{~nm}$ 的基态吸收峰。但是, ${ }^{5} \mathrm{D}_{4}$ 能级与 $4 \mathrm{f}^{7} 5 \mathrm{~d}^{1}$ 最低的 ${ }^{9} \mathrm{D}$ 能级间跃迁属于双重禁戒跃迁, 因此激发态吸收概 率非常低。

表 3 Dy : YAP 晶体与 $D y^{3+}$ 掺杂其他基质的光谱参数

Table 3 Spectroscopic parameters of $\mathrm{Dy}^{3+}$ doped YAP and other crystals

\begin{tabular}{|c|c|c|c|c|c|}
\hline Host & $\sigma_{\mathrm{abs}} /\left(\times 10^{-21}, \mathrm{~cm}^{2}\right)$ & $\beta / \%$ & $\sigma_{\mathrm{em}} /\left(\times 10^{-20}, \mathrm{~cm}^{2}\right)$ & $\tau_{\mathrm{f}} / \mu \mathrm{s}$ & Ref. \\
\hline YAG & 1.6 & 50.96 & 1.50 & 376 & [64] \\
\hline $\operatorname{YAl}\left(\mathrm{BO}_{3}\right)_{4}$ & - & 65.90 & 1.90 & 520 & {$[65]$} \\
\hline $\mathrm{LiLuF}_{4}$ & - & 65.40 & 1.02 & 582 & {$[66]$} \\
\hline $\mathrm{Lu}_{2} \mathrm{SiO}_{4}$ & - & 61.0 & 0.74 & 509 & {$[67]$} \\
\hline $\mathrm{KY}_{3} \mathrm{~F}_{10}$ & - & 59.8 & 0.83 & 440 & {$[66]$} \\
\hline $\mathrm{Li}_{2} \mathrm{Gd}_{4}\left(\mathrm{MoO}_{4}\right)_{7}$ & $\begin{array}{c}\sigma: 2.5 \\
\pi: 4.4 \\
a: 0.743\end{array}$ & $\begin{array}{c}\sigma: 72.0 \\
\pi: 71.9 \\
a: 88.5\end{array}$ & $\begin{array}{l}\sigma: 1.45 \\
\pi: 1.34 \\
a: 0.298\end{array}$ & 139 & {$[68]$} \\
\hline YAP & $\begin{array}{l}b: 0.690 \\
c: 0.870\end{array}$ & $\begin{array}{l}b: 88.7 \\
c: 87.8\end{array}$ & $\begin{array}{l}b: 0.450 \\
c: 0.452\end{array}$ & 185 & This work \\
\hline
\end{tabular}

表 4 掺 $\mathrm{Dy}^{3+}$ 激光晶体在可见波段的激光输出

Table 4 Laser output of $\mathrm{Dy}^{3+}$ doped some common laser crystal

\begin{tabular}{ccccccccc}
\hline Crystal & $\lambda_{\text {em }} / \mathrm{nm}$ & Polarization & Laser transition & $\eta_{\text {slope }} / \%$ & $P_{\text {out }} / \mathrm{mW}$ & $P_{\text {thr }} / \mathrm{mW}$ & Type & Year \\
\hline Dy, Tb : LLF & 574 & $\sigma$ & ${ }^{4} \mathrm{~F}_{9 / 2} \rightarrow{ }^{6} \mathrm{H}_{13 / 2}$ & 13 & 55 & 320 & $\mathrm{CW}$ & $2014^{[72]}$ \\
Dy,Eu : YLF & 574 & $\sigma$ & ${ }^{4} \mathrm{~F}_{9 / 2} \rightarrow{ }^{6} \mathrm{H}_{13 / 2}$ & 10 & 47 & 244 & $\mathrm{CW}$ & $2016^{[17]}$ \\
& 578 & $\sigma$ & ${ }^{4} \mathrm{~F}_{9 / 2} \rightarrow{ }^{6} \mathrm{H}_{13 / 2}$ & 4 & 17 & 188 & Self-pulsed & $2016^{[17]}$ \\
Dy : LLF & 661 & $\pi$ & ${ }^{4} \mathrm{~F}_{9 / 2} \rightarrow{ }^{6} \mathrm{H}_{11 / 2}$ & 2 & 4 & 143 & Self-pulsed & $2016^{[17]}$ \\
Dy : YAG & 583 & - & ${ }^{4} \mathrm{~F}_{9 / 2} \rightarrow{ }^{6} \mathrm{H}_{13 / 2}$ & 12 & 150 & - & Self-pulsed & $2012^{[64]}$ \\
Dy : ZnWO & 575 & $E / / b$ & ${ }^{4} \mathrm{~F}_{9 / 2} \rightarrow{ }^{6} \mathrm{H}_{13 / 2}$ & 13 & 110 & 550 & $\mathrm{CW}$ & $2017^{[71]}$ \\
\hline
\end{tabular}


$\mathrm{Tb}^{3+}$ 主要有 $544 、 590$ 和 $625 \mathrm{~nm}$ 三组发光峰, 分 别属于绿光、黄橙光和红光, 发射截面约 $10^{-21} \mathrm{~cm}^{2}$ 。 在基质选择方面, 氧化物材料也适宜作为 $\mathrm{Tb}^{3+}$ 的基 质材料 ${ }^{[15]}$ 。首先, 单禁戒跃迁 $\mathrm{Tb}^{3+}$ 的能量要比 $\operatorname{Pr}^{3+}$ 更大, 其次在高声子能量的氧化物中, $\mathrm{Tb}^{3+}$ 的 ${ }^{5} \mathrm{D}_{4}$ 能 级也不易发生非辐射弛豫。

$\mathrm{Tb}$ : YAP 晶体偏振吸收光谱如图 11 所示。 $\mathrm{Tb}^{3+}$ 的吸收主要来自于 ${ }^{7} \mathrm{~F}_{6}$ 基态能级分别到 ${ }^{5} \mathrm{G}_{2}+{ }^{5} \mathrm{~L}_{6}$ 、 ${ }^{5} \mathrm{G}_{4}+{ }^{5} \mathrm{~L}_{9} 、{ }^{5} \mathrm{G}_{5}+{ }^{5} \mathrm{D}_{2} 、{ }^{5} \mathrm{~L}_{10} 、{ }^{5} \mathrm{D}_{3}+{ }^{5} \mathrm{G}_{6} 、{ }^{5} \mathrm{D}_{4} 、{ }^{7} \mathrm{~F}_{0} 、{ }^{7} \mathrm{~F}_{1}$ 、 ${ }^{7} \mathrm{~F}_{2}$ 和 ${ }^{7} \mathrm{~F}_{3}$ 能级的吸收跃迁。由于近红外区域的跃迁 属于自旋允许跃迁, 所以对应的吸收截面较大。然 而, 吸收波长处于可见区域, 与 $\mathrm{InGaN}$ 的发射波长 匹配度较好的 ${ }^{7} \mathrm{~F}_{6} \rightarrow{ }^{5} \mathrm{D}_{4}$ 跃迁属于自旋禁戒跃迁, 其 相应的吸收截面较低 ${ }^{[73]}$ 。 $10^{-22} \mathrm{~cm}^{2}$ 量级的吸收截面 相对较低, 可通过高掺杂浓度来弥补, 且不会发生 ${ }^{5} \mathrm{D}_{4}$ 能级浓度猝灭, 其掺杂浓度可高达 $10^{21} \mathrm{~cm}^{-3}$ 。综上 所述, Tb : YAP 适合使用蓝光 InGaN LD 进行泵浦。

图 12 所示为 $\mathrm{Tb}: \mathrm{YAP}$ 晶体的偏振荧光光谱。 三个发射跃迁 ${ }^{5} \mathrm{D}_{4} \rightarrow{ }^{7} \mathrm{~F}_{5} 、{ }^{7} \mathrm{~F}_{4} 、{ }^{7} \mathrm{~F}_{3}$ 对应的发射峰分别 位于 544、 590、 $624 \mathrm{~nm}$ 。其中, $544 \mathrm{~nm}$ 的发光峰 最强, 在 $a 、 b 、 c$ 三个偏振方向的值分别为 $6.37 \times 10^{-22} 、 6.61 \times 10^{-22} 、 11.12 \times 10^{-22} \mathrm{~cm}^{2}$ 。

值得注意的是, $\mathrm{Tb}$ : YAP 晶体的黄光发射截面 大于 $\mathrm{TbP}_{5} \mathrm{O}_{14}(\mathrm{TPP})\left(587 \mathrm{~nm}, 1.0 \times 10^{-22} \mathrm{~cm}^{2}\right) 、 \mathrm{TbLiP}_{4} \mathrm{O}_{12}$ (TLP) $\left(588 \mathrm{~nm}, 1.0 \times 10^{-22} \mathrm{~cm}^{2}\right)$ 和 $\mathrm{TbAl}_{3}\left(\mathrm{BO}_{3}\right)_{4}(\mathrm{TAB})$ $\left(592 \mathrm{~nm}, 1.0 \times 10^{-22} \mathrm{~cm}^{2}\right)^{[75]}$ 。结果表明 $\mathrm{Tb}$ : YAP 晶体 是黄光激光输出非常有潜力的备选材料。

$\mathrm{Tb}^{3+}$ 掺杂不同材料的吸收波长 $\lambda_{\mathrm{abs}}$ 、吸收截面 $\sigma_{\mathrm{abs}} 、{ }^{5} \mathrm{D}_{4} \rightarrow{ }^{7} \mathrm{~F}_{4}$ 跃迁发射波长 $\lambda_{\mathrm{em}}$ 、发射截面 $\sigma_{\mathrm{em}}$ 和苂 光寿命 $\tau_{\mathrm{f}}$ 如表 5 所示。 $\mathrm{Tb}: \mathrm{YAP}$ 的 $\sigma_{\mathrm{abs}} 、 \sigma_{\mathrm{em}}$ 和 $\tau_{\mathrm{f}}$ 值 较大。发射截面 $\sigma_{\mathrm{em}}$ 和寿命 $\tau$ 的乘积与激光阈值成反 比, $\mathrm{Tb}$ ：YAP 黄光跃迁在 $a 、 b 、 c$ 偏振方向分别为 $2.96 \times$ $10^{-22} \mathrm{~cm}^{2} \cdot \mathrm{ms} 、 4.70 \times 10^{-22} \mathrm{~cm}^{2} \cdot \mathrm{ms} 、 4.56 \times 10^{-22} \mathrm{~cm}^{2} \cdot \mathrm{ms}$ 。
其中, $b$ 偏振对应的数值大于 TPP、TLP、TAB、 PZABP 以及 LBTAF，小于 LLF。也就是说, Tb : YAP 的激光阈值大于 $\mathrm{Tb}: \mathrm{LLF}$ ，但是小于上表材料中其 他材料, 有利于低阈值的黄光激光输出。值得关注 的是, 虽然 $\mathrm{Tb}$ : YAP 晶体的黄光发射截面相对较大, 但是 $10^{-22} \mathrm{~cm}^{2}$ 量级仍然较小。可结合 $\mathrm{Tb}^{3+}$ 能够实现 高浓度掺杂的特性, 实现高效的泵浦光吸收和黄光 发射。以上数据均表明 $\mathrm{Tb}$ : YAP 晶体可成为一种有 前景的黄光激光晶体。

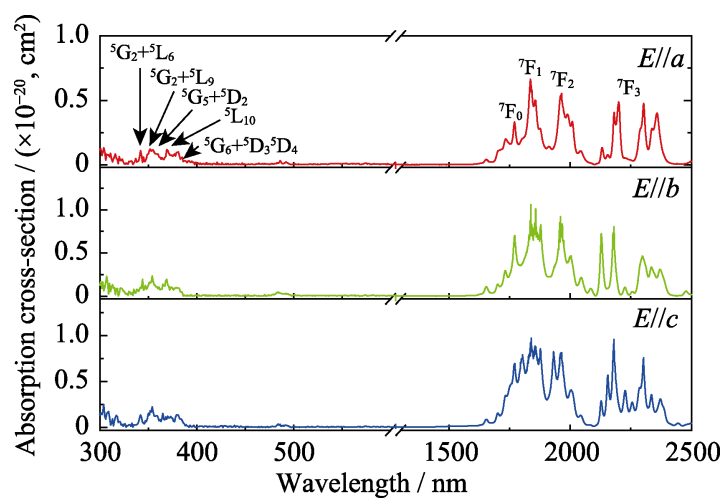

图 $11 \mathrm{~Tb}$ : YAP 晶体的偏振吸收光谱 ${ }^{[74]}$

Fig. 11 Polarized absorption spectra of Tb : YAP crystal ${ }^{[74]}$

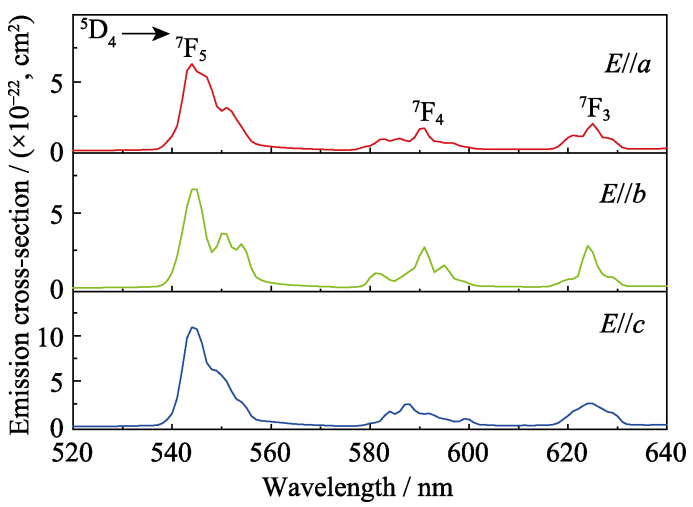

图 $12 \mathrm{~Tb}$ : YAP 晶体的偏振荧光光谱 ${ }^{[74]}$

Fig. 12 Polarized fluorescence spectra of Tb : YAP crystal ${ }^{[74]}$

表 $5 \mathrm{~Tb}$ : YAP 晶体与 $\mathbf{T b}^{3+}$ 掺杂其他材料的光谱参数

Table 5 Spectroscopic parameters of $\mathrm{Tb}^{3+}$ doped YAP and other crystals

\begin{tabular}{cccccccc}
\hline Host & $\lambda_{\mathrm{abs}} / \mathrm{nm}$ & $\sigma_{\mathrm{abs}} /\left(\times 10^{-22}, \mathrm{~cm}^{2}\right)$ & $\lambda_{\mathrm{em}} / \mathrm{nm}$ & $\sigma_{\mathrm{em}} /\left(\times 10^{-22}, \mathrm{~cm}^{2}\right)$ & $\tau_{\mathrm{f}} / \mathrm{ms}$ & Ref. \\
\hline LLF & 488.8 & 3.0 & 585 & $\sim 11$ & 4.8 & {$[76]$} \\
TPP & 485 & 1.3 & 587 & 1.0 & 3.4 & {$[75]$} \\
TLP & 487 & 2.2 & 588 & 1.0 & 3.7 & {$[75]$} & {$[75]$} \\
TAB & 483 & 3.6 & 592 & 1.0 & 0.8 & 0.42 & {$[77]$} \\
PZABP & - & - & 582 & 585 & 0.58 & 1.27 & {$[78]$} \\
\hline LBTAF & - & - & 5.3 & 590 & 1.72 & & This work \\
YAP & $E / / a$ & 486 & 5.2 & 591 & 2.73 & & 1.72 \\
\hline
\end{tabular}




\section{2 掺 $\mathbf{T b}^{3+}$ 激光晶体的激光特性}

$\mathrm{Tb}^{3+}$ 可见光激光在 1967 年首次实现, 因为其截 面较小, 实现激光输出的报道相对较少 ${ }^{[79]}$ 。第一个 $\mathrm{Tb}^{3+}$ 激光输出是 $\mathrm{Ar}^{+}$激光洜浦 $\mathrm{Tb}^{3+}$ 掺杂氟化物光纤, 发射波长为 $543 \mathrm{~nm}$, 斜率效率 $4 \%{ }^{[80]}$ 。表 6 列出了 近年来掺 $\mathrm{Tb}^{3+}$ 激光晶体在室温下可见波段的连续激 光输出。最近, 使用 $2 \omega$-OPSL 泵浦, 成功实现了 $\mathrm{Tb}^{3+}$ 掺杂氟化物材料在绿光和黄光波段的激光输出, 由于样品长度和掺杂浓度限制, 最终洜浦光的吸收 效率低于 $30 \%$ 。21 mm 长 $\mathrm{Tb}: \mathrm{LiLuF}_{4}$ 首次实现绿光 输出功率超过 $1 \mathrm{~W}$, 光转化效率达到 $40 \%$, 对应斜 率效率高达 52\% ${ }^{[17]}$ 。 $\mathrm{Tb}: \mathrm{LiYF}_{4}$ 首次实现黄光 $587 \mathrm{~nm}$ 激光运转, 斜率效率 $22 \%$, 最高输出功率 $71 \mathrm{~mW}^{[81]}$ 。 随后, $\mathrm{Tb}: \mathrm{KY}_{3} \mathrm{~F}_{10}$ 中也实现了连续黄光和绿光激光 输出。相比于复杂的倍频方法实现黄光激光运转, 这种方法更加便捷。

应当注意的是, 虽然 $\mathrm{Tb}^{3+}$ 跃迁截面较低, 输出 耦合镜透过率需要达到 $1 \%$, 甚至更高, 但是可以通 过 $\mathrm{Tb}^{3+}$ 的高浓度掺杂来实现高效率激光运转。 $\mathrm{TbF}_{3}$ 中 $100 \% \mathrm{~Tb}^{3+}$ 掺杂成功实现激光运转, 很好地证明 了其可行性 ${ }^{[81]}$ 。

\section{4 掺 $\mathrm{Sm}^{3+}$ 激光晶体}

\section{1 掺 $\mathrm{Sm}^{3+}$ 激光晶体的光谱特性}

$\mathrm{Sm}^{3+}$ 最大的吸收峰在 $400 \mathrm{~nm}$ 处, 吸收截面达到 $10^{-20} \mathrm{~cm}^{2}$ 。因此非常适宜使用 $\mathrm{GaN}$ 激光二极管进行 泵浦。比如, $480 \mathrm{~nm}$ 处吸收峰的吸收截面约为 $10^{-21} \mathrm{~cm}^{2}$ 。由于 $\mathrm{Sm}^{3+}$ 的 $4 \mathrm{f}$ 电子层能级结构复杂, 易 产生交叉驰豫而导致荧光寿命发生猝灭, 因此其粒 子数浓度不应大于 $4 \times 10^{20} \mathrm{~cm}^{-3[17]}$ 。 $\mathrm{Sm}^{3+}$ 的 $5 \mathrm{~d}$ 能级 位置相比 $\operatorname{Pr}^{3+}$ 要高, 即使在晶格场较强的材料中, 激发态吸收发生的概率也很低。 ${ }^{4} \mathrm{G}_{5 / 2}$ 能级到邻近下
能级的带隙高达 $6000 \mathrm{~cm}^{-1}$, 因此 $\mathrm{Sm}^{3+}$ 总体上对基 质声子能量的要求并不苛刻, 氧化物材料完全可以 用作其基质材料。由于 $\mathrm{Sm}^{3+}$ 复杂的能级结构, 在不 合适的材料中容易发生激发态吸收、能量上转换和 交叉驰豫。

$\mathrm{Sm}^{3+}$ 发射光谱中, 从 ${ }^{4} \mathrm{G}_{5 / 2}$ 到 ${ }^{6} \mathrm{H}_{\mathrm{j}}$ 能级在可见波 段的大部分发光峰属于禁戒跃迁。最主要的发光波 段是 $560 \mathrm{~nm}$ 绿光、 $600 \mathrm{~nm}$ 橙光、 $650 \mathrm{~nm}$ 红光和 $700 \mathrm{~nm}$ 深红光, 对应的能级跃迁分别为 ${ }^{4} \mathrm{G}_{5 / 2} \rightarrow$ ${ }^{6} \mathrm{H}_{5 / 2} 、{ }^{4} \mathrm{G}_{5 / 2} \rightarrow{ }^{6} \mathrm{H}_{7 / 2} 、{ }^{4} \mathrm{G}_{5 / 2} \rightarrow{ }^{6} \mathrm{H}_{9 / 2}$ 和 ${ }^{4} \mathrm{G}_{5 / 2} \rightarrow{ }^{6} \mathrm{H}_{11 / 2}$ 。橙 光发射截面接近 $10^{-21} \mathrm{~cm}^{2}$, 绿光仅有 $10^{-22} \mathrm{~cm}^{2}$ 。 ${ }^{4} \mathrm{G}_{5 / 2}$ 能级的苂光寿命较长, 可以达到 $\mathrm{ms}$ 量级。

图 13 为 $\mathrm{Sm}$ : YAP 晶体的偏振吸收光谱图。其 中一些吸收由于相互作用强而难以完全分离 ${ }^{[84]}$ 。在 可见光区域, 三种偏振下 $\mathrm{Sm}^{3+}$ 的最强吸收峰均位于 $409 \mathrm{~nm}$ 处, $a 、 b$ 和 $c$ 偏振对应的吸收截面分别为 $0.25 \times 10^{-20} 、 0.67 \times 10^{-20}$ 和 $0.86 \times 10^{-20} \mathrm{~cm}^{2}$ ，半高宽 FWHM 分别为 $8.4 、 10.2$ 和 $9.4 \mathrm{~nm}$ 。 Sm: YAP 晶体的 吸收截面与 $\mathrm{Sm}$ : BYF 的吸收截面 $\left(0.55 \times 10^{-20} \mathrm{~cm}^{2}\right)^{[85]}$ 相当。大的吸收截面和带宽非常适合使用 GaN/InGaN LD 进行泵浦。

$\mathrm{Sm}$ : YAP 晶体的偏振苂光光谱如图 14 所示。 在可见光区, $\mathrm{Sm}^{3+}$ 四个主要发射带分别为 ${ }^{4} \mathrm{G}_{5 / 2} \rightarrow$ ${ }^{6} \mathrm{H}_{5 / 2} 、{ }^{4} \mathrm{G}_{5 / 2} \rightarrow{ }^{6} \mathrm{H}_{7 / 2} 、{ }^{4} \mathrm{G}_{5 / 2} \rightarrow{ }^{6} \mathrm{H}_{9 / 2}$ 和 ${ }^{4} \mathrm{G}_{5 / 2} \rightarrow{ }^{6} \mathrm{H}_{11 / 2}$ 。由 于 YAP 晶体的各向异性 ${ }^{[21]}$, 可以看到发射光谱呈现 出很强的偏振依赖性, 对于 $a 、 b$ 和 $c$ 偏振, 各谱峰 发射强度差距较大。

其中, ${ }^{4} \mathrm{G}_{5 / 2} \rightarrow{ }^{6} \mathrm{H}_{7 / 2}$ 跃迁的 $b$ 偏振发射截面最大, 为 $1.01 \times 10^{-21} \mathrm{~cm}^{2}$ 。能级辐射寿命与发射截面的乘积 作为表征激光运转阈值相关的重要参数, 与激光阈 值成反比例关系。Sm : YAP 晶体在 $b$ 偏振方向, ${ }^{4} \mathrm{G}_{5 / 2}$ 能级的辐射寿命与 ${ }^{4} \mathrm{G}_{5 / 2} \rightarrow{ }^{6} \mathrm{H}_{7 / 2}$ 跃迁对应发射截面的 乘积为 $2.38 \times 10^{-21} \mathrm{~cm}^{2} \cdot \mathrm{ms}$, 低于 $\mathrm{Sm}: \mathrm{LiYF}_{4}$ 晶体 ${ }^{[86]}$ 和 $\mathrm{Sm}: \mathrm{LiLuF}_{4}$ 晶体 ${ }^{[87]}$, 但是大于 $\mathrm{Sm}: \mathrm{LiNbO}_{3}$ 晶体 ${ }^{[84]}$ 。

表 6 掺 $\mathbf{T b}^{3+}$ 激光晶体室温下的可见波段连续激光输出

Table 6 Laser output of $\mathbf{T b}^{3+}$ doped some common laser crystal

\begin{tabular}{|c|c|c|c|c|c|c|c|c|}
\hline Crystal & $\lambda_{\mathrm{em}} / \mathrm{nm}$ & Polarization & Laser transition & $\eta_{\text {slope }} / \%$ & $P_{\text {out }} / \mathrm{mW}$ & $P_{\mathrm{thr}} / \mathrm{mW}$ & Pump source & Year \\
\hline \multirow{2}{*}{$\mathrm{Tb}:$ YLF } & 542 & $\sigma$ & ${ }^{5} \mathrm{D}_{4} \rightarrow{ }^{7} \mathrm{~F}_{5}$ & 55 & 158 & 8 & $2 \omega-\mathrm{OPSL}$ & \\
\hline & 587 & $\pi$ & ${ }^{5} \mathrm{D}_{4} \rightarrow{ }^{7} \mathrm{~F}_{4}$ & 22 & 71 & 32 & $2 \omega-\mathrm{OPSL}$ & \\
\hline \multirow{2}{*}{$\mathrm{Tb}: \mathrm{LLF}$} & 542 & $\sigma$ & ${ }^{5} \mathrm{D}_{4} \rightarrow{ }^{7} \mathrm{~F}_{5}$ & 52 & 1130 & 32 & $2 \omega-\mathrm{OPSL}$ & \\
\hline & 587 & $\pi$ & ${ }^{5} \mathrm{D}_{4} \rightarrow{ }^{7} \mathrm{~F}_{4}$ & 14 & 82 & 107 & $2 \omega-\mathrm{OPSL}$ & $2016^{[81]}$ \\
\hline \multirow{2}{*}{$\mathrm{Tb}: \mathrm{KYF}$} & 545 & $\sigma$ & ${ }^{5} \mathrm{D}_{4} \rightarrow{ }^{7} \mathrm{~F}_{5}$ & 34 & 793 & 25 & $2 \omega-\mathrm{OPSL}$ & \\
\hline & 584 & $\pi$ & ${ }^{5} \mathrm{D}_{4} \rightarrow{ }^{7} \mathrm{~F}_{4}$ & 5 & 18 & 38 & $2 \omega-\mathrm{OPSL}$ & \\
\hline $\mathrm{Tb}: \mathrm{BLuF}$ & 546 & $\sigma$ & ${ }^{5} \mathrm{D}_{4} \rightarrow{ }^{7} \mathrm{~F}_{5}$ & 46 & 270 & 18 & $2 \omega-\mathrm{OPSL}$ & \\
\hline $\mathrm{Tb}: \mathrm{CaF}_{2}$ & 541 & - & ${ }^{5} \mathrm{D}_{4} \rightarrow{ }^{7} \mathrm{~F}_{5}$ & 48 & 103 & $\sim 34$ & $2 \omega-\mathrm{OPSL}$ & $2017^{[82]}$ \\
\hline
\end{tabular}




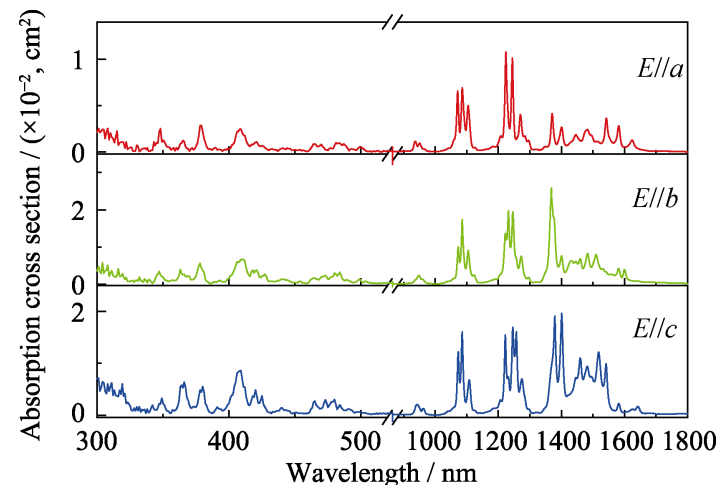

图 $13 \mathrm{Sm}$ : YAP 晶体的偏振吸收谱图 ${ }^{[83]}$

Fig. 13 Polarized absorption spectra of Sm : YAP crystal ${ }^{[83]}$

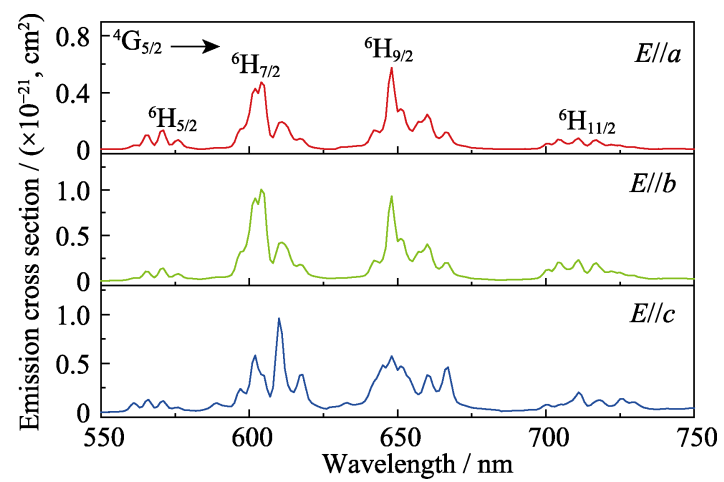

图 $14 \mathrm{Sm}$ : YAP 晶体的偏振苂光光谱 ${ }^{[83]}$

Fig. 14 Polarized fluorescence spectra of Sm : YAP crystal ${ }^{[83]}$

说明 $\mathrm{Sm}$ : YAP 晶体 ${ }^{4} \mathrm{G}_{5 / 2} \rightarrow{ }^{6} \mathrm{H}_{7 / 2}$ 跃迁对应的激光阈 值高于 $\mathrm{Sm}: \mathrm{LiYF}_{4}$ 和 $\mathrm{Sm}: \mathrm{LiLuF}_{4}$, 但低于 $\mathrm{Sm}: \mathrm{LiNbO}_{3}$ 。众所周知, 氟化物材料的机械硬度和 导热系数等物理特性相对较差, 而激光运转过程中, 材料良好的热学性能和机械性能是十分重要的。与 同体系的 YAG 晶体相似, Sm : YAP 晶体拥有高的热 导率和机械强度, 可以更好地保证激光输出的稳定 性 ${ }^{[84]}$ 。以上结果表明 $\mathrm{Sm}$ : YAP 晶体是红橙色激光 输出非常有希望的备选材料。
表 7 对 $\mathrm{Sm}^{3+}$ 掺杂 YAP 与其他已实现激光输出 基质的光谱参数进行了比较。 $\mathrm{Sm}$ ：YAP 在 $400 \mathrm{~nm}$ 附近吸收峰带宽更大，使用 $\mathrm{InGaN}$ 激光二极管进行 泵浦更方便。Sm：YAP 在 $600 \mathrm{~nm}$ 附近发光峰的发 射截面与 $\mathrm{Sm}: \mathrm{SrAl}_{12} \mathrm{O}_{19} 、 \mathrm{Sm}: \mathrm{LiLuF}_{4}$ 和 $\mathrm{Sm}: \mathrm{LiYF}_{4}$ 材料相差无几。这三种基质是目前 $\mathrm{Sm}^{3+}$ 掺杂实现激 光输出最具代表性的基质材料。Sm：YAP 晶体中的 荧光寿命相对较小, 这一定程度上归因于 $\mathrm{Sm}^{3+}$ 掺杂 浓度偏高。苂光寿命的增大可以通过优化 $\mathrm{Sm}^{3+}$ 掺杂 浓度来实现。综上所述, Sm : YAP 在 $600 \mathrm{~nm}$ 附近红 橙激光输出方面非常有可能成为潜在的替代材料。

\section{2 掺 $\mathrm{Sm}^{3+}$ 激光晶体的激光特性}

1979 年, $\mathrm{Sm}^{3+}$ 掺杂 $\mathrm{TbF}_{3}$ 首次实现激光输出 ${ }^{[89]}$ 。 $\mathrm{Tb}^{3+}$ 高浓度掺杂提升了对百浦光的有效吸收, 此过 程很大程度上依赖于 $\mathrm{Sm}^{3+}$ 上能级 ${ }^{4} \mathrm{G}_{5 / 2}$ 与 $\mathrm{Tb}^{3+}$ 上能 级 ${ }^{5} \mathrm{D}_{4}$ 间的能量传递。后来, 蓝光 $\mathrm{Ar}^{+}$激光器泵浦 $\mathrm{Sm}^{3+}$ 掺杂玻璃光纤成功获得了 $651 \mathrm{~nm}$ 处 $10 \mathrm{~mW}$ 连 续激光输出 ${ }^{[90]}$ 。 2015 年, 首次使用蓝光半导体百浦 源激发 $\mathrm{Sm}^{3+}$ 掺杂 $\mathrm{LiLuF}_{4}$ 在 $606 \mathrm{~nm}$ 处成功实现激光 输出, 输出功率近 $100 \mathrm{~mW}$, 斜率效率 $15 \%$ 。同时, $\mathrm{Sm}, \mathrm{Mg}$ : $\mathrm{SrAl}_{12} \mathrm{O}_{19}$ 晶体成功实现 $703 \mathrm{~nm}$ 处 $50 \mathrm{~mW}$ 激光输出, 以及 $593 \mathrm{~nm}$ 处 $10 \mathrm{~mW}$ 激光输出, 斜率效 率为 $1 \%{ }^{[88]}$ 。但是, $\mathrm{Sm}^{3+}$ 激光材料橙光的效率明显要 低, 这可能是 $\mathrm{Sm}^{3+}$ 复杂的能级结构造成的。并且其 吸收截面偏小, 䈋浦光吸收效率低于 $25 \%$, 可以通 过使用 $\mathrm{GaN}$ 泵浦源来进一步提升其效率。

\section{5 掺 $\mathbf{H o}^{3+}$ 激光晶体}

\section{1 掺 $\mathrm{Ho}^{3+}$ 激光晶体的光谱特性}

$\mathrm{Ho}^{3+}$ 的主要发射波长位于 $2 \mu \mathrm{m}$ 附近 ${ }^{[91]}$ 。在 $450 \mathrm{~nm}$ 波长，吸收截面达到 $10^{-20} \mathrm{~cm}^{2}$ ，适合使用 $\mathrm{InGaN}$ 激 光二极管泵浦; 在绿光波段的吸收截面更大，适合

表 $7 \mathrm{Sm}$ : YAP 晶体与 $\mathrm{Sm}^{3+}$ 掺杂其他材料的光谱参数

Table 7 Spectroscopic parameters of $\mathrm{Sm}^{3+}$ doped YAP and other crystals

\begin{tabular}{|c|c|c|c|c|c|c|c|c|}
\hline Sample & $\lambda_{\mathrm{abs}} / \mathrm{nm}$ & $\sigma_{\mathrm{abs}} /\left(\times 10^{-20}, \mathrm{~cm}^{2}\right)$ & $\mathrm{FWHM}_{\mathrm{abs}} / \mathrm{nm}$ & $\lambda_{\mathrm{em}} / \mathrm{nm}$ & $\sigma_{\mathrm{em}} /\left(\times 10^{-21}, \mathrm{~cm}^{2}\right)$ & $\mathrm{FWHM}_{\mathrm{em}} / \mathrm{nm}$ & $\tau_{\mathrm{f}} / \mathrm{ms}$ & Ref. \\
\hline $\mathrm{Sm}: \mathrm{SrAl}_{12} \mathrm{O}_{19}$ & $400(\sigma)$ & $8.5(\sigma)$ & - & $593(\sigma)$ & $1.2(\sigma)$ & - & 3.4 & {$[88]$} \\
\hline \multirow{2}{*}{$\mathrm{Sm}: \mathrm{LiLuF}_{4}$} & $401(\sigma)$ & $1.04(\sigma)$ & $2.3(\sigma)$ & \multirow{2}{*}{$606(\pi)$} & \multirow{2}{*}{$1.3(\pi)$} & $7.5(\sigma)$ & \multirow{2}{*}{4.8} & \multirow{2}{*}{ [87-88] } \\
\hline & $401(\pi)$ & $1.51(\pi)$ & $3.1(\pi)$ & & & $9.5(\pi)$ & & \\
\hline \multirow{2}{*}{$\mathrm{Sm}: \mathrm{LiYF}_{4}$} & $401(\sigma)$ & $0.72(\sigma)$ & $3(\sigma)$ & $597(\sigma)$ & $0.679(\sigma)$ & $7.5(\sigma)$ & \multirow{2}{*}{4.8} & \multirow{2}{*}[86]{} \\
\hline & $401(\pi)$ & $1.37(\pi)$ & $2(\pi)$ & $605(\pi)$ & $1.039(\pi)$ & $9.5(\pi)$ & & \\
\hline \multirow{3}{*}{ Sm : YAP } & $409(E / / a)$ & $0.25(E / / a)$ & $8.4(E / / a)$ & $604(E / / a)$ & $0.47(E / / a)$ & $6.29(E / / a)$ & \multirow{3}{*}{0.59} & \multirow{3}{*}{ This work } \\
\hline & $409(E / / b)$ & $0.67(E / / b)$ & $10.2(E / / b)$ & $604(E / / b)$ & $1.01(E / / b)$ & $6.32(E / / b)$ & & \\
\hline & $409(E / / c)$ & $0.86(E / / c)$ & $9.4(E / / c)$ & $610(E / / c)$ & $0.96(E / / c)$ & $3.26(E / / c)$ & & \\
\hline
\end{tabular}


使用倍频 $\mathrm{Nd}^{3+}$ 激光作为洜浦源。在这两种情况下, 都存在 $\mathrm{Ho}^{3+}$ 的 ${ }^{5} \mathrm{~F}_{3}$ 能级到 ${ }^{5} \mathrm{~S}_{2}$ 和 ${ }^{5} \mathrm{~F}_{4}$ 能级间的非辐射 弛豫现象 ${ }^{[92]}$ 。这些能级理论上均可作为四能级跃迁 机制的上激光发射能级, 而对于激光下能级去激发 的主要影响能级是 ${ }^{5} \mathrm{I}_{7}$ 能级。此能级属于 $2 \mu \mathrm{m}$ 激光 跃迁的上激光能级, 寿命较长, 可达 $10 \mathrm{~ms}^{[91]}$ 。因此 粒子数容易在此能级发生阻塞, 抑制与上能级间的 粒子数反转。因此, 比较可行的可见波段激光跃迁 是 ${ }^{5} \mathrm{~S}_{2},{ }^{5} \mathrm{~F}_{4} \rightarrow{ }^{5} \mathrm{I}_{8}$ 的绿光跃迁。在 $\mathrm{Ho}: \mathrm{LiLuF}_{4}$ 中, 此跃 迁在 $535 \mathrm{~nm}$ 处对应的发射截面就达 $2 \times 10^{-20} \mathrm{~cm}^{2}$ 。 然而, 此波长需要的激光阈值相对较高, 跃迁到斯 塔克分裂高能级的截面较小 $\left(\sim 0.5 \times 10^{-20} \mathrm{~cm}^{2}\right)$ 。并且, 其上能级的苂光寿命一般短于 $1 \mathrm{~ms}$ 。有研究发现, 即使在已实现激光输出的低声子能量基质 $\mathrm{LiLuF}_{4}$ 中, 声子能量仍会通过 ${ }^{5} \mathrm{~F}_{5}$ 能级发生非辐射弛豫。因此, 只有声子能量非常低的基质材料, 比如卤化物适合 作为 $\mathrm{Ho}^{3+}$ 掺杂实现可见激光输出的基质材料 ${ }^{[93-94]}$ 。

由于 $4 \mathrm{f}^{9} 5 \mathrm{~d}^{1}$ 高的能级位置, 激发态吸收对于 $\mathrm{Ho}^{3+}$ 来说是很难发生的。但是, 一些交叉驰豫是不 可避免的, 离子掺杂浓度不能大于 $10^{19} \mathrm{~cm}^{-3}$, 即在 大多数基质材料中不要超过 $1 \mathrm{at} \%$ 。

\section{2 掺 $\mathbf{H o}^{3+}$ 激光晶体的激光特性}

掺杂 $\mathrm{Ho}^{3+}$ 实现可见波段激光输出在早期吸引了 一定的关注。比如闪光灯泵浦 $\mathrm{Ho}: \mathrm{CaF}_{2}{ }^{[95]}$, 在低温 下实现 ${ }^{5} \mathrm{~F}_{4},{ }^{5} \mathrm{~S}_{2} \rightarrow{ }^{5} \mathrm{I}_{8}$ 跃迁绿光激光输出, 使用闪光灯 洜浦 $\mathrm{Ho}_{\mathrm{N}} \mathrm{LiYF}_{4}$ 在室温下实现 ${ }^{5} \mathrm{~F}_{4},{ }^{5} \mathrm{~S}_{2} \rightarrow{ }^{5} \mathrm{I}_{7}$ 跃迁脉冲 激光输出 ${ }^{[96]}$ 。最近, 首次使用蓝光 $2 \omega$-OPSL 葲浦 $\mathrm{Ho}: \mathrm{LaF}_{3}$ 和 $\mathrm{Ho}: \mathrm{LiLuF}_{4}$ 实现可见波段激光输出 ${ }^{[97]}$ 。 $\mathrm{Ho}: \mathrm{LaF}_{3}$ 晶体在 $549 \mathrm{~nm}$ 波长处输出功率不到 $10 \mathrm{~mW}$, 斜率效率为 $0.4 \%$ 。这两种材料的激光阈值较高, 不 同点在于 $\mathrm{LaF}_{3}$ 的声子能量更低, 所以 ${ }^{5} \mathrm{~F}_{4},{ }^{5} \mathrm{~S}_{2}$ 能级的 非辐射弛豫概率更低。然而, 由于 ${ }^{5} \mathrm{I}_{7}$ 能级长寿命引 起的粒子数阻塞, 即使在 $\mathrm{Ho}: \mathrm{LaF}_{3}$ 材料中, 激光输 出形式也仅限于自脉冲激光。此外, 在声子能量更 低的 Ho: ZBLAN 光纤中, 使用 $643 \mathrm{~nm}$ 上转换洜浦 方式, 成功实现 $40 \mathrm{~mW}$ 绿光 $(550 \mathrm{~nm})$ 连续激光输出, 斜率效率大于 $20 \%{ }^{[98]}$ 。由于泵浦光耦合进光纤的效 率较低以及上转换过程存在较大的复杂性, 在蓝光 波段直接百浦可能会实现 Ho：ZBLAN 光纤绿光更 高的斜率效率。

\section{6 掺 $\mathbf{E r}^{3+}$ 激光晶体}

\section{1 掺 $\mathbf{E r}^{3+}$ 激光晶体的光谱特性}

除了比较熟知的 1.5 和 $3 \mu \mathrm{m}$ 红外波段激光跃
迁 ${ }^{[99-101]}, \mathrm{Er}^{3+}$ 也存在可见激光跃迁，代表性的是通 过上转换方法实现绿光激光发射 ${ }^{[102-103]}{ }_{\circ} \mathrm{Er}^{3+}$ 在 405 、 450 和 $485 \mathrm{~nm}$ 处存在吸收峰, 允许蓝光 $\mathrm{LD}$ 的直接 泵浦。前两个波长的吸收截面一般为 $10^{-21} \mathrm{~cm}^{2}$, 在 $485 \mathrm{~nm}$ 附近, $\mathrm{Er}: \mathrm{LiLuF}_{4}$ 晶体吸收截面达到 $1.5 \times$ $10^{-20} \mathrm{~cm}^{2}$, 与 $2 \omega$-OPSL 泵浦源发射波长相吻合, 且 此处吸收截面比 $970 \mathrm{~nm}$ 处的吸收截面高 $50 \%$ 左右。 由于 $\mathrm{Er}^{3+}$ 复杂的能级结构, 在蓝光洜浦下, 激发态 吸收过程很容易发生，但目前还没有明确证实。在 一些基质材料中, 上能级 ${ }^{4} \mathrm{~S}_{3 / 2}$ 的苂光寿命普遍为 $100 \mu \mathrm{s}$ 左右 ${ }^{[102]}$ 。 $\mathrm{Er}^{3+}$ 在 $550 \mathrm{~nm}$ 处发射截面约为 $10^{-20} \mathrm{~cm}^{2}$, 高于 $\operatorname{Pr}^{3+}$ 之外的其他可见波段稀土离子 ${ }^{[104]}$ 。 并且, $\mathrm{Er}^{3+}$ 距离 $4 \mathrm{f}^{10} 5 \mathrm{~d}^{1}$ 能级的带隙较大, 不容易产生 $5 \mathrm{~d}$ 激发态吸收 ${ }^{[105]}$ 。

由于浓度增加会引起苂光猝灭, $\mathrm{Er}^{3+}$ 最大的掺 杂浓度不应超过 $2 \times 10^{20} \mathrm{~cm}^{-3}$ 。激光上能级 ${ }^{4} \mathrm{~S}_{3 / 2}$ 到下 能级 ${ }^{4} \mathrm{~F}_{9 / 2}$ 带隙为 $3000 \mathrm{~cm}^{-1}$, 与 $\mathrm{Pr}^{3+}$ 相接近, 因此在 低声子能量氟化物中基本不发生非辐射弛豫, 但在 氧化物材料中明显观测到 ${ }^{4} \mathrm{~S}_{3 / 2}$ 能级寿命缩短, 甚至 达到一个量级。这可能是 ${ }^{4} \mathrm{~S}_{3 / 2}$ 与长寿命 ${ }^{4} \mathrm{I}_{13 / 2}$ 能级间 发生交叉驰豫所引起的, 除了引起寿命猝灭, 还有 可能导致激光运转的不稳定。此外, 氧化物材料中 强晶格场引起的能级繴裂会降低 ${ }^{4} \mathrm{~S}_{3 / 2}$ 到 ${ }^{4} \mathrm{~F}_{9 / 2}$ 能级间 的能量差, 增大非辐射弛豫的几率, 进而造成 ${ }^{4} \mathrm{I}_{13 / 2}$ 发生粒子数阻塞。结果表明, 高声子能量的氧化物 材料不适合 $\mathrm{Er}^{3+}$ 掺杂实现可见光激光输出。

\section{2 掺 $\mathrm{Er}^{3+}$ 激光晶体的激光特性}

直接百浦 $\mathrm{Er}^{3+}$ 掺杂材料获得可见光激光的报道 很少，目前主要是基于上转换泵浦来实现绿光激光 输出 ${ }^{[102]}$ 。然而, 研究发现在蓝光泵浦波段, 激光阈 值是相对较低的 ${ }^{[106]}$ 。之前, 这种方法的洜浦源主要 使用染料激光器, 脉冲宽度 $50 \mathrm{~ns}$, 重复频率为 $10 \mathrm{~Hz}$, 使用其百浦 $\mathrm{Er}: \mathrm{LiYF}_{4}$ 样品, 在 $486 \mathrm{~nm}$ 处吸收葲浦 能量达到 $600 \mu \mathrm{J}$ 时, 实现 $551 \mathrm{~nm}$ 处能量为 $35 \mu \mathrm{J}$ 的 激光输出 ${ }^{[107]}$ 。在以上实验中, 斜率效率都不超过 $6 \%$ 。虽然 $\mathrm{Er}^{3+}$ 可见波段上能级的苂光寿命较短，但 在氧化物 YAG 和 $\mathrm{LuAG}$ 中也达到了激光阈值。

为了证明 $\mathrm{Er}^{3+}$ 掺杂材料实现蓝光波段连续激光 输出的可行性, Kränkel 等 ${ }^{[17]}$ 使用 $2 \omega$-OPSL(最大输 出功率为 $4 \mathrm{~W}$ ) 原浦源洜浦 $\mathrm{Er}: \mathrm{LiLuF}_{4}$, 首次实现 $552 \mathrm{~nm}$ 波长处输出功率为 $33 \mathrm{~mW}$ 、斜率效率 $9 \%$ 的 连续激光输出。低效率可能是由于吸收峰带宽较窄, 吸收效率较低 ${ }^{[17]}$ 。更高功率的激光输出可以通过加 长晶体长度, 提高冷却系统效率等方法实现。 


\section{7 掺 $\mathbf{E u}^{3+}$ 激光晶体}

\section{1 掺 $\mathbf{E u}^{3+}$ 激光晶体的光谱特性}

通常 $\mathrm{Eu}^{3+}$ 的发射谱线处于红色光区, 这些谱线 对应于 $4 \mathrm{f}$ 电子组态内从激发态 ${ }^{5} \mathrm{D}_{0}$ 能级到 ${ }^{7} \mathrm{~F}_{J}(J=0,1$, $2,3,4,5,6)$ 能级的跃迁发射 ${ }^{[108]}$ 。由于 ${ }^{5} \mathrm{D}_{0}$ 能级不被 晶体场分裂 $(J=0)$, 发射跃迁的分裂是由 ${ }^{7} \mathrm{~F}_{J}$ 能级的 晶体场分裂引起的。除了这些谱线外, 有时还可以 观察到来自较高的 ${ }^{5} \mathrm{D}_{1} 、{ }^{5} \mathrm{D}_{2}$ 甚至 ${ }^{5} \mathrm{D}_{3}$ 能级的发射。

当稀土离子所占据晶格格位具有反演对称性时, 4f 电子组态能级间的电子跃迁发射属于宇称选律严 格禁戒的电偶极跃迁, 它们只能作为强度很弱的磁 偶极跃迁 $(\Delta J=0, \pm 1)$ 或电子振动电偶极跃迁发生。如 果稀土离子所处的格位没有反演对称性, 那么晶体 场奇次项可以将相反宇称态混合到组态 4f 能级中, 此时电偶极跃迁不再是严格禁戒的, 光谱中就会出 现受迫电偶极跃迁的谱线。某些跃迁(即 $\Delta J=0, \pm 2$ 的 跃迁)对此效应极为灵敏。即便是 $\mathrm{Eu}^{3+}$ 所处的格位仅 稍微偏离反演对称性, 此跃迁发射在光谱中也占主 导地位。受迫电偶极跃迁发射必须满足以下两个条 件, 即在 $\mathrm{Eu}^{3+}$ 的晶体学格位上不存在反演对称中心, 而且电荷迁移跃迁处于低能位置。 $\mathrm{Eu}^{3+}$ 所处的晶体 场格位仅稍微偏离反演对称性, 其谱线常以 ${ }^{5} \mathrm{D}_{0} \rightarrow$ ${ }^{7} \mathrm{~F}_{2}$ (约 $610 \mathrm{~nm}$ 处)受迫电偶极跃迁发射红光为主, 而 $\mathrm{Eu}^{3+}$ 处于有严格反演对称中心的格位时, 将以允许 的 ${ }^{5} \mathrm{D}_{0} \rightarrow{ }^{7} \mathrm{~F}_{1}$ 磁偶极跃迁发射黄光。

\section{2 掺 $\mathbf{E u}^{3+}$ 激光晶体的激光特性}

$\mathrm{Eu}^{3+}$ 作为激活剂, 在稀土离子中发光性能是优 异的。它能发射单色性好, 量子效率高的红光。 $\mathrm{Eu}^{3+}$ 的红光发射现已被广泛用于彩色电视、平板显示、 阴极射线管 $(\mathrm{CRT})$ 及三基色荧光粉中。目前, 对 $\mathrm{Eu}^{3+}$ 的可见光研究大多集中在苂光粉方面。其中 $\mathrm{YVO}_{4}: \mathrm{Eu}^{3+}, \mathrm{Y}_{2} \mathrm{O}_{3}: \mathrm{Eu}^{3+}$ 等是最有代表性的红光发
射荧光体。而对 $\mathrm{Eu}^{3+}$ 激光晶体研究较少。2015 年 Dashkevich 等 ${ }^{[109]}$ 使用 $\mathrm{LD}$ 泵浦 $\mathrm{Eu}: \mathrm{KGd}\left(\mathrm{WO}_{4}\right)_{2}$ 晶体, 实现 $702 \mathrm{~nm}$ 处 $5.4 \mathrm{~mW}$ 的连续激光运转。最近 Demesh 等 ${ }^{[110]}$ 采用钛宝石泵浦 $\mathrm{Eu}$ : Y LF, 获得了 $702 \mathrm{~nm}$ 处 $15 \mathrm{~mW}$, 斜率效率 $4.6 \%$ 的连续激光输出。

\section{8 总结与展望}

图 15 为使用蓝光半导体二极管泵浦稀土离子 掺杂激光材料在可见波段激光输出功率的汇总。可 以看出, 在可见波段激光输出中, $\mathrm{Pr}^{3+}$ 离子输出功率 相对较高, 在大多数波长输出功率已超过 $1 \mathrm{~W}$; 此 外左图中激光输出的功率较高, 这与氟化物材料声 子能量较低, 有利于减少无辐射弛豫能量损失, 从 而获得较高激光效率有关 ${ }^{[109]}$ 。 $\operatorname{Pr}^{3+}$ 是目前发展比较 广泛的激活离子, 吸收 $\left(10^{-19} \mathrm{~cm}^{2}\right.$ 量级 $)$ 和发射 $\left(10^{-20} \sim 10^{-21} \mathrm{~cm}^{2}\right.$ 量级)截面大, 斜效率超过 $70 \%$, 输 出功率大于 $4 \mathrm{~W}$ 。目前在氟化物中激光最大输出功 率要大于氧化物, 在蓝、绿、橙、红和深红波段都 有激光输出报道。

考虑到综合的物化性能, 除了 Pr: YLF、 $\operatorname{Pr}$ ：LLF 等晶体外, 522、621 nm 波段的 $\operatorname{Pr}$ : YAP 和 宽发射(超快可见光)的 Pr: SRA 等晶体尤其值得关 注。 $\operatorname{Pr}^{3+}$ 的缺点是: (1)不能直接发射黄光。(2)上激光 能级很容易无辐射弛豫到 ${ }^{1} \mathrm{D}_{2}$ 能级, 所以基质声子 能量不能太大。(3)上激光能级与 $4 \mathrm{f}^{1} 5 \mathrm{~d}^{1}$ 带隙较窄, 容易发生激发态吸收跃迁，长期以来研究基质多是 晶格场较弱的氟化物晶体, 但是, 结合本文所提出 的 $\operatorname{Pr}^{3+}$ 掺杂氧化物材料判据, 最近一些低声子能量、 高配位氧化物材料也实现了激光输出, 非常适合进 一步研究。

$\mathrm{Dy}^{3+}$ 易发生交叉驰豫, 掺杂浓度不宜过高。吸 收截面 $\left(10^{-21} \mathrm{~cm}^{2}\right.$ 量级)小, 激光效率低, 最高斜率效 率为 $13 \%$ 。其 $4 \mathrm{f}^{8} 5 \mathrm{~d}^{1}$ 能带位置较高, 所以非常适合
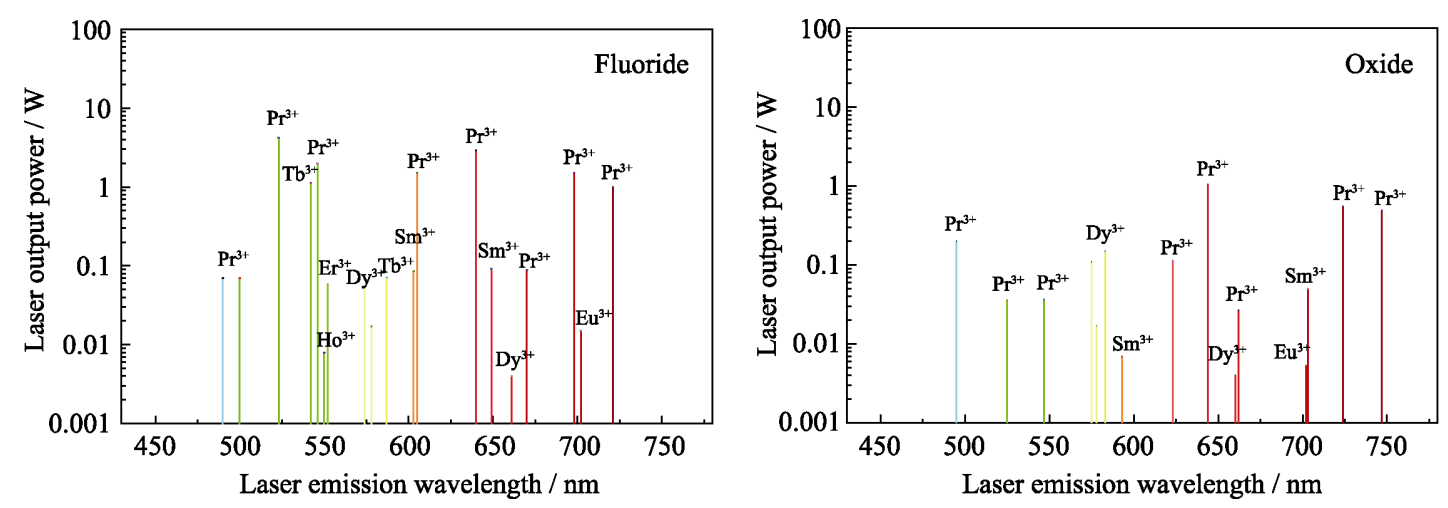

图 $15 \mathrm{Pr}^{3+} 、 \mathrm{Dy}^{3+} 、 \mathrm{~Tb}^{3+} 、 \mathrm{Sm}^{3+} 、 \mathrm{Ho}^{3+} 、 \mathrm{Er}^{3+} 、 \mathrm{Eu}^{3+}$ 掺杂激光材料的输出功率与发射波长关系图

Fig. 15 Output power $v s$ laser wavelength of $\mathrm{Pr}^{3+}, \mathrm{Dy}^{3+}, \mathrm{Tb}^{3+}, \mathrm{Sm}^{3+}, \mathrm{Ho}^{3+}, \mathrm{Er}^{3+}, \mathrm{Eu}^{3+}$ doped some common crystals 
氧化物基质。 $\mathrm{Dy}^{3+}$ 发展的一个重要原因是可以发射 黄光, 通过共掺去激活离子 $\left(\mathrm{Tb}^{3+}\right.$ 或 $\left.\mathrm{Eu}^{3+}\right)$ 缩短下能 级寿命, 减少粒子数阻塞, 提高黄光的激光输出功 率和效率。

$\mathrm{Tb}^{3+}$ 吸收截面 $\left(10^{-22} \mathrm{~cm}^{2}\right.$ 量级)更小, 可以通过高 掺杂 $(\sim 10 \mathrm{at} \%)$ 或离子敏化来弥补, 具有长的苂光寿 命, 适合脉冲调 $\mathrm{Q}$ 激光运转。 $\mathrm{Tb}^{3+}$ 的一个优点是可 以发射绿、黄、橙、红光 ${ }^{[111]}$, 绿光激光输出功率超 过 $1 \mathrm{~W}$, 斜率效率达 $58 \%$, 黄光的对应效率可以达 到 $22 \%$ 。其自旋禁戒跃迁能量比 $\operatorname{Pr}^{3+}$ 高, 适合氧化 物激光运转。因而 $\mathrm{Tb}^{3+}$ 氧化物晶体非常有发展潜力, 可见光的倍频也是以后的一个发展方向。

$\mathrm{Sm}^{3+}$ 易发生交叉驰豫, 掺杂浓度不宜过高, 因 为其 $5 \mathrm{~d}$ 能级位置高, 所以即使在强晶体场、大声子 能量基质中也可以。但是 $\mathrm{Sm}^{3+}$ 发射波长与 $\mathrm{Pr}^{3+}$ 比较 接近, 面临 $\mathrm{Pr}^{3+}$ 的竞争。 $\mathrm{Ho}^{3+}$ 可见激光要求低声子能 量基质, $\mathrm{Er}^{3+}$ 能级结构复杂, 且发射波长与 $\mathrm{Pr}^{3+}$ 接 近。 $\mathrm{Eu}^{3+}$ 适合氧化物掺杂, 掺杂浓度较高, 但其主要 红光发射波长与 $\operatorname{Pr}^{3+}$ 接近。

\section{参考文献:}

[1] 徐军. 激光材料科学与技术前沿. 上海: 上海交通大学出版 社, 2007.

[2] 徐军. 稀土激光晶体材料及其应用. 北京: 科学出版社, 2016 .

[3] 徐军. 新型激光晶体材料及其应用. 北京: 科学出版社, 2016.

[4] ZHAO LING, YAO YI, ZHAO YANG, et al. All-solid-state dual end pumped $\mathrm{YVO}_{4}: \mathrm{Nd} / \mathrm{LBO}$ blue laser with $21.8 \mathrm{~W}$ output power at $457 \mathrm{~nm}$. Opt. Spectrosc., 2014, 116(3): 470-472.

[5] KANTOLA EMMI, LEINONEN TOMI, RANTA SANNA, et al. High-efficiency $20 \mathrm{~W}$ yellow VECSEL. Opt. Express, 2014, 22(6): 6372-6380.

[6] YU HAO-HAI, ZONG NAN, PAN ZHONG-BEN, et al. Efficient high-power self-frequency-doubling $\mathrm{Nd}$ :GdCOB laser at 545 and $530 \mathrm{~nm}$. Opt. Lett., 2011, 36(19): 3852-3854.

[7] FANG QIAN-NAN, LU DA-ZHI, YU HAO-HAI, et al. Selffrequency-doubled vibronic yellow $\mathrm{Yb}$ :YCOB laser at the wavelength of $570 \mathrm{~nm}$. Opt. Lett., 2016, 41(5): 1002-1005.

[8] PASCHOTTA RUDIGE, MOORE NICK, CLARKSON W. ANDREW, et al. $230 \mathrm{~mW}$ of blue light from a thulium-doped upconversion fiber laser. IEEE. J. Sele. Top. Quant., 1997, 3(4): $1100-1102$.

[9] SANDROCK T, SCHEIFE H, HEUMANN E, et al. High-power continuous-wave upconversion fiber laser at room temperature. Opt. Lett., 1997, 22(11): 808-810.

[10] ROTH PRTER W, MACLEAN ALEXANDER J, BURNS DAVID, et al. Directly diode-laser-pumped Ti:sapphire laser. Opt. Lett., 2009, 34(21): 3334-3336.

[11] GÜREL K, WITTWER V J, HOFFMANN M, et al. Green-diodepumped femtosecond Ti: sapphire laser with up to $450 \mathrm{~mW}$ average power. Opt. Express, 2015, 23(23): 30043-30048.

[12] NAKAMURA SHUJI, SENOH MASAYUKI, NAGAHAMA SHIN-ICHI, et al. InGaN-based multi-quantum-well-structure laser diodes. Jpn. J. Appl. Phys., 1996, 35(1B): 74-76.

[13] KUZNETSOV M, HAKIMI F, SPRAGUE R, et al. High-power
( $>0.5-\mathrm{W}$ CW) diode-pumped vertical-external-cavity surfaceemitting semiconductor lasers with circular $\mathrm{TEM}_{00}$ beams. IEEE Photonics Tech., 1997, 9(8): 1063-1065.

[14] MCINERNEY J G, MOORADIAN A, LEWIS A, et al. Highpower surface emitting semiconductor laser with extended vertical compound cavity. Electron. Lett., 2003, 39(6): 523-525.

[15] REICHERT FABIAN, MARZAHL DANIEL-TIMO, METZ PHILIP-WERNER, et al. Efficient laser operation of diodepumped $\mathrm{Pr}^{3+}, \mathrm{Mg}^{2+}: \mathrm{SrAl}_{12} \mathrm{O}_{19}$. Appl. Phys. B, 2014, 116(1): 109113.

[16] MALINOWSKI M, JOUBERT M F, MAHIOU R, et al. Visible laser emission of $\mathrm{Pr}^{3+}$ in various hosts. J. Phys. IV, 1994, 4(C4): 541-544.

[17] KRÄNKEL CHRISTIAN, MARZAHL DANIEL-TIMMO, MOGLIA FRANCESCA, et al. Out of the blue: semiconductor laser pumped visible rare-earth doped lasers. Laser. Photon. Rev., 2016, 10(4): 548-568.

[18] LIU BIN, SHI JIAO-JIAO, WANG QING-GUO, et al. Crystal growth, polarized spectroscopy and Judd-Ofelt analysis of Pr:YAlO ${ }_{3}$. J. Lumin., 2018, 196: 76-80.

[19] REICHERT FABINA, MARAZAHL DANIEL-TIMO, HUBER-GUNTER. Spectroscopic characterization and laser performance of $\mathrm{Pr}, \mathrm{Mg}: \mathrm{CaAl}_{12} \mathrm{O}_{19}$. J. Opt. Soc. Am. B, 2014, 31(2): 349-354.

[20] FECHNER M, REICHERT F, HANSEN NO, et al. Crystal growth, spectroscopy, and diode pumped laser performance of Pr,Mg: $\mathrm{SrAl}_{12} \mathrm{O}_{19}$. Appl. Phys. B, 2011, 102(4): 731-735.

[21] DANGER T, BLECKMANN A, HUBER G. Stimulated emission and laser action of $\mathrm{Pr}^{3+}$-doped $\mathrm{YAlO}_{3}$. Appl. Phys. B, 1994, 58(5): 413-420.

[22] WANG YAN, LI JIAN-FU, YOU ZHEN-YU, et al. Spectroscopic properties of $\mathrm{Pr}^{3+}: \mathrm{Gd}_{3} \mathrm{Ga}_{5} \mathrm{O}_{12}$ crystal. J. Alloy. Compd., 2010, 502(1): 184-189.

[23] CORNACCHIA F, LIETO A-DI, TONELLi M, et al. Efficient visible laser emission of GaN laser diode pumped Pr-doped fluoride scheelite crystals. Opt. Express, 2008, 16(20): 15932-15941.

[24] RECHICHERT F, MOGLIA F, MARZAHL D T, et al. Diode pumped laser operation and spectroscopy of $\operatorname{Pr}^{3+}: \mathrm{LaF}_{3}$. Opt. Express, 2012, 20(18): 20387-20395.

[25] KHIARI S, VELAZQUEZ M, MONCORGE R, et al. Red-luminescence analysis of $\operatorname{Pr}^{3+}$ doped fluoride crystals. $\mathrm{J}$. $\mathrm{Al}$ loy. Compd., 2008, 451: 128-131.

[26] HAKIM R, DAMAK K, TONCELLI A, et al. Growth, optical spectroscopy and Judd-Ofelt analysis of Pr-doped $\mathrm{BaY}_{2} \mathrm{~F}_{8}$ monocrystals. J. Lumin., 2014, 143: 233-240.

[27] MARZAHL DANIEL-TIMO, REICHERT FABIN, FECHNER MATHIES, et al. Laser Operation and Spectroscopy of $\operatorname{Pr}^{3+}$ : $\mathrm{LaMgAl}_{11} \mathrm{O}_{19}$. 5th Eps-Qeod Europhoton Conference, 2012.

[28] SATTAYAPORN S, LOISEAU P, AKA G, et al. Crystal growth, spectroscopy and laser performances of $\mathrm{Pr}^{3+}: \mathrm{Sr}_{0.7} \mathrm{La}_{0.3} \mathrm{Mg}_{0.3} \mathrm{Al}_{11.7} \mathrm{O}_{19}$ (Pr:ASL) . Opt. Express, 2008, 26(2): 1278-1289.

[29] HOMMERICH U, BROWN E, AMEDZAKE P, et al. Mid-infrared $(4.6 \mu \mathrm{m})$ emission properties of $\mathrm{Pr}^{3+}$ doped $\mathrm{KPb}_{2} \mathrm{Br}_{5}$. J. Appl. Phys., 2006, 100: 113507.

[30] SOJKA L, TANG A, FURNISS D. et al. Broadband, mid-infrared emission from $\mathrm{Pr}^{3+}$ doped GeAsGaSe chalcogenide fiber, optically clad. Opt. Mater., 2014, 36: 1076-1082.

[31] WALSH BRIAN-M, HOMMERICH UWE, YOSHIKAWA AKIRA, et al. Mid-infrared spectroscopy of Pr-doped materials. J. Lumin., 2018, 197: 349-353.

[32] ZANDI BAHRAM, MERKEL LARRY-D, GRUBER JOHN-B, et al. Optical spectra and analysis for $\operatorname{Pr}^{3+}$ in $\mathrm{SrAl}_{12} \mathrm{O}_{19}$. J. Appl. 
Phys., 1997, 81(3): 1047-1054.

[33] MOOS H-WARREN. Spectroscopic relaxation processes of rare earth ions in crystals. J. Lumin., 1970, 1(2): 106-121.

[34] DORENBOS P. 5d-level energies of $\mathrm{Ce}^{3+}$ and the crystalline environment. I. Fluoride compounds. Phys. Rev. B, 2000, 62(23): $15640-15649$.

[35] LAROCHE M, BRAUD ALAIN, GIARD, et al. Spectroscopic investigations of the $4 \mathrm{f} 5 \mathrm{~d}$ energy levels of $\mathrm{Pr}^{3+}$ in fluoride crystals by excited-state absorption and two-step excitation measurements. J. Opt. Soc. Am. B, 1999, 16(12): 2269-2277.

[36] LAROCHE MATHIEU, DOUALAN JEAN-LOUIS, GIRARD SYLVAIN, et al. Experimental and theoretical investigations of the $4 \mathrm{f}^{2} \backslash 4 \mathrm{f} 5 \mathrm{~d}$ ground-state and excited-state absorption spectra of $\mathrm{Pr}^{3+}$ in $\mathrm{LiYF}_{4}$. J. Opt. Soc. Am. B, 2000, 17(7): 1291-1303.

[37] LAYNE C B, LOWDERMILK W H, WECER M J. Multiyhonon relaxation of rare-earth ions in oxide glasses. Phys. Rev., 1977, 16(1): $11-20$.

[38] SCHUURAMANS M F H, VAN DIJK J M F. On radiative and non-radiative decay times in the weak coupling limit. Phy. B, 1984, 123(2): 131-155.

[39] NIKL MATIN, YOSHIKAWA AKIRA. Recent R\&D trends in inorganic single-crystal scintillator materials for radiation detection. Adv. Optical Mater, 2015, 3(4): 463-481.

[40] CHEUNG Y M, GAYEN S K. Excited-state absorption in $\mathrm{Pr}^{3+}: \mathrm{Y}_{3} \mathrm{Al}_{5} \mathrm{O}_{12}$. Phys. Rev. B, 1994, 49(21): 14827-14835.

[41] SOLOMON R, MUELLER L. Stimulated emission at $598.5 \mathrm{~nm}$ from $\mathrm{Pr}^{3+}$ in $\mathrm{LaF}_{3}$. Appl. Phys. Lett., 1963, 3(8): 135-137.

[42] SANDROCK T, DANGER T, HEUMANN E, et al. Efficient continuous wave-laser emission of $\mathrm{Pr}^{3+}$-doped fluorides at room temperature. Appl. Phys. B, 1994, 58(2): 149-151.

[43] SANDROCK T, HEUMANN E, HUBER G, et al. Continuouswave $\mathrm{Pr}, \mathrm{Yb}: \mathrm{LiYF}_{4}$ Upconversion Laser in the Red Spectral Range at Room Temperature. Advanced Solid State Lasers, Optical Society of America, 1996.

[44] METZ P-W, HASSE K, PARISI D, et al. Continuous-wave $\mathrm{Pr}^{3+}: \mathrm{BaY}_{2} \mathrm{~F}_{8}$ and $\mathrm{Pr}^{3+}: \mathrm{LiYF}_{4}$ lasers in the cyan-blue spectral region. Opt. Lett., 2014, 39(17): 5158-5161.

[45] METZ PHILIP-WERNER, REICHERT FABIAN, MOGLIA FRANCESCA, et al. High-power red, orange, and green $\mathrm{Pr}^{3+}: \mathrm{LiYF}_{4}$ lasers. Opt. Lett., 2014, 39(11): 3193-3196.

[46] TANAKA HIROKI, KANNARI FUMIHIKO. Power Scaling of Continuous-wave Visible $\mathrm{Pr}^{3+}$ :YLF Laser End-pumped by High Power Blue Laser Diodes. OSA Laser Congress, 2017.

[47] LUO SAI-YU, YAN XI-GUN, CUI QIN, et al. Power scaling of blue-diode-pumped Pr:YLF lasers at 523.0, 604.1, 606.9, 639.4, 697.8 and $720.9 \mathrm{~nm}$. Opt. Commun., 2016, 380: 357-360.

[48] CORNACCHIA F, RICHTER A, HEUMANN E, et al. Visible laser emission of solid state pumped $\mathrm{LiLuF}_{4}: \mathrm{Pr}^{3+}$. Opt. Express, 2007, 15: 992-1002.

[49] SOTTILE ALBERTO, PARISI DANIELA, TONELLI MAURO. Multiple polarization orange and red laser emissions with Pr:BaY ${ }_{2} \mathrm{~F}_{8}$. Opt. Express, 2014, 22(11): 13784-13791.

[50] METZ PHILIP W, MULLER SEBASTIAN, REICHERT FABIAN, et al. Wide wavelength tunability and green laser operation of diode-pumped $\operatorname{Pr}^{3+}: \mathrm{KY}_{3} \mathrm{~F}_{10}$. Opt. Express, 2013, 21(25): 31274-31281.

[51] YU HAO, JIANG DA-PENG, TANG FEI, et al. Enhanced photoluminescence and initial red laser operation in $\mathrm{Pr}: \mathrm{CaF}_{2}$ crystal via co-doping $\mathrm{Gd}^{3+}$ ions. Mater. Lett., 2017, 206: 140-142.

[52] SATTAYAPORN S, LOISEAU P, AKA G, et al. Crystal growth, spectroscopy and laser performances of $\operatorname{Pr}^{3+}: \mathrm{Sr}_{0.7} \mathrm{La}_{0.3} \mathrm{Mg}_{0.3} \mathrm{Al}_{11.7} \mathrm{O}_{19}$ (Pr:ASL). Opt. Express, 2018, 26(2): 1278-1289.
[53] FIBRICH MARTIN, SULC JAN, JELINKOVA HELENA. Pr: $\mathrm{YAlO}_{3}$ laser generation in the green spectral range. Opt. Lett., 2013, 38(23): 5024-5027.

[54] FIBRICH, JELINKOVA H, SULC J, et al. $\mathrm{Pr}_{\mathrm{YAlO}} \mathrm{Y}_{3}$ microchip laser at $662 \mathrm{~nm}$. Laser Phys. Lett., 2011, 8(2): 116-119.

[55] FIBRICH M, SULC J, JELINKOVA H. Power-scaling of a $\mathrm{Pr}: \mathrm{YAlO}_{3}$ microchip laser operating at $747 \mathrm{~nm}$ wavelength at room temperature. Laser Phys. Lett., 2014, 11(10): 105802-1-4.

[56] REICHERT F, CALMANOT, MULLER S, et al. Efficient visible laser operation of $\mathrm{Pr}, \mathrm{Mg}: \mathrm{SrAl}_{12} \mathrm{O}_{19}$ channel waveguides. Opt. Lett., 2013, 38(15): 2698-2701.

[57] REICHERT F, CALMANO T, MÜLLER S, et al. Visible Laser Operation of $\mathrm{Pr}, \mathrm{Mg}: \mathrm{SrAl}_{12} \mathrm{O}_{19}$ Waveguides. The European Conference on Lasers and Electro-Optics, 2013.

[58] KRANKEL CHRISTIAN. Rare-earth-doped sesquioxides for diode-pumped high-power lasers in the 1-, 2-, and 3- $\mu \mathrm{m}$ spectral range. IEEE Journal of Selected Topics in Quantum Electronics, 2015, 21(1): 250-262.

[59] DIALlO P T, BOUTIAUD P, MAHIOU R, et al. Red luminescence in $\operatorname{Pr}^{3+}$-doped calcium titanates. Phys. Status Solidi., 1997, 160(1): 255-263.

[60] LIU BIN, SHI JIAO-JIAO, WANG QING-GUO, et al. Crystal growth and yellow emission of Dy: $\mathrm{YAlO}_{3}$. Opt. Mater., 2017, 72: 208-213.

[61] NING KAI-JIE, HE XIAO-MING, ZHANG LIAN-HAN, et al. Spectroscopic characteristics of $\mathrm{GdVO}_{4}: \mathrm{Dy}^{3+}$ crystal. Opt. Mater., 2014, 37: 745-749.

[62] YANG FU-GUI, TU CHAO-YANG, WANG HONG-YAN, et al. Growth and spectroscopy of $\mathrm{Dy}^{3+}$ doped in $\mathrm{ZnWO}_{4}$ crystal. Opt. Mater, 2007, 29(12): 1861-1865.

[63] LUPEI A, LUPEI V, GHEORGHE C, et al. Spectroscopic characteristics of $\mathrm{Dy}^{3+}$ doped $\mathrm{Y}_{3} \mathrm{Al}_{5} \mathrm{O}_{12}$ transparent ceramics. J. Appl. Phys., 2011, 110(8): 083120-1-9.

[64] BOWMAN R, O'CONNOR S, CONDON N J. Diode pumped yellow dysprosium lasers. Opt. Express, 2012, 20(12): 12906-1 2911.

[65] RYBA-ROMANOWSKI W, DOMINIAK-DZIK G, SOLARZ P, et al. Transition intensities and excited state relaxation dynamics of $\mathrm{Dy}^{3+}$ in crystals and glasses: a comparative study. Opt. Mater., 2009, 31(11): 1547-1554.

[66] BIGOTTA STEFANO, TONELLI M, CAVALLI E, et al. Optical spectra of $\mathrm{Dy}^{3+}$ in $\mathrm{KY}_{3} \mathrm{~F}_{10}$ and $\mathrm{LiLuF}_{4}$ crystalline fibers. $J$. $\mathrm{Lu}$ min., 2010, 130(1): 13-17.

[67] DOMINIAK-DZIK G, RYBA-ROMANOWSKI W, LISIECKI R, et al. Dy-doped $\mathrm{Lu}_{2} \mathrm{SiO}_{5}$ single crystal: spectroscopic characteristics and luminescence dynamics. Appl. Phys. B, 2009, 99(1/2): 285-297.

[68] ZHOU WEI-WEI, WEI BO, ZHAO WANG, et al. Intense yellow emission in $\mathrm{Dy}^{3+}$-doped $\mathrm{LiGd}\left(\mathrm{MoO}_{4}\right)_{2}$ crystal for visible lasers. Opt. Mater, 2011, 34(1): 56-60.

[69] MALINOWSKI M, MYZIAK P, PIRAMIDOWICZ R, et al. Spectroscopic and laser properties of $\mathrm{LiNbO}_{3}: \mathrm{Dy}^{3+}$ crystals. Acta Phys. Pol. A, 1996, 1(90): 181-189.

[70] LIMPERT J, ZELLMER H, RIEDEL P, et al. Laser Oscillation in Yellow and Blue Spectral Range in $\mathrm{Dy}^{3+}$ :ZBLAN. Lasers and Electro-Optics, 2001. CLEO'01. Technical Digest, 2001: 353354.

[71] XIA ZHONG-CHAO, YANG FU-GUI, QIAO LIANG, et al. End pumped yellow laser performance of $\mathrm{Dy}^{3+}: \mathrm{ZnWO}_{4}$. Opt. Commun., 2017, 387: 357-360.

[72] BOLOGNESI GIACOMO, PARIS DANIELA, CALONICO DAVIDE, et al. Yellow laser performance of $\mathrm{Dy}^{3+}$ in co-doped 
Dy,Tb:LiLuF 4 . Opt. Lett., 2014, 39(23): 6628-6631.

[73] LOIKO PAVEL, MATEOS XAVIER, DUNINA ELENA, et al. Judd-Ofelt modelling and stimulated-emission cross-sections for $\mathrm{Tb}^{3+}$ ions in monoclinic $\mathrm{KYb}\left(\mathrm{WO}_{4}\right)_{2}$ crystal. J. Lumin., 2017, 190: $37-44$

[74] LIU BIN, SHI JIAO-JIAO, WANG QING-GUO, et al. Crystal growth, polarized spectroscopy and Judd-Ofelt analysis of Tb: $\mathrm{YAlO}_{3}$. Spectrochim. Acta A, 2018, 200: 58-62.

[75] COLAK S, ZWICKER W K. Transition rates of $\mathrm{Tb}^{3+}$ in $\mathrm{TbP}_{5} \mathrm{O}_{14}$, $\mathrm{TbLiP}_{4} \mathrm{O}_{12}$, and $\mathrm{TbAl}_{3}\left(\mathrm{BO}_{3}\right)_{4}$ : an evaluation for laser applications. J. Appl. Phys., 1983, 54(5): 2156-2166.

[76] METZ P W, MARZAHL D T, MAJID A, et al. High Power Continuous Wave Visible $\mathrm{Tb}^{3+}: \mathrm{LiLuF}_{4}$ Laser. Advanced Solid State Lasers Conference, 2015: ATu1A.1.

[77] KESAVULU C R, IDAALME SILVA ANIELLE-CHRISTINE, DOUSTI M-R, et al. Concentration effect on the spectroscopic behavior of $\mathrm{Tb}^{3+}$ ions in zinc phosphate glasses. J. Lumin., 2015, 165: 77-84.

[78] JAMALAIAH B C, SURESH KUMAR, MOHAN BABU A, et al. Study on spectroscopic and fluorescence properties of $\mathrm{Tb}^{3+}$-doped LBTAF glasses. Physica B Condens. Matter, 2009, 404(14/15): 2020-2024.

[79] ANDREEV S I, BEDILOV M R, KARAPETYAN G O, et al. Stimulated radiation of glass activated by terbium. Sov. J. Opt. Tech., 1967, 34: 819.

[80] YAMASHITA TATSUYA, OHISHI YASUTAKE. Amplification and lasing characteristics of $\mathrm{Tb}^{3+}$-doped fluoride fiber in the 0.54 m band. Jpn. J. Appl. Phys., 2007, 46(11): 991-993.

[81] METZ P W, MARZAHL D T, MAJID A, et al. Efficient continuous wave laser operation of $\mathrm{Tb}^{3+}$-doped fluoride crystals in the green and yellow spectral regions. Laser \& Photonics Reviews, 2016, 10(2): 335-344.

[82] PHILIP WERNER METZ, DANIEL-TIMO MARZAHL, GÜNTER HUBER, et al. Performance and wavelength tuning of green emitting terbium lasers. Opt. Express, 2017, 17(25): 5716-5724.

[83] 刘斌. 可见波段稀土离子掺杂激光晶体的生长和性能研究. 上海: 同济大学博士学位论文, 2018.

[84] DOMINIAK-DZIK GRAZYNA. $\mathrm{Sm}^{3+}$-doped $\mathrm{LiNbO}_{3}$ crystal, optical properties and emission cross-sections. J. Alloy. Compd., 2005, 391(1/2): 26-32.

[85] PUGH-THOMAS D. Spectroscopic properties and Judd-Ofelt analysis of $\mathrm{BaY}_{2} \mathrm{~F}_{8}: \mathrm{Sm}^{3+}$. J. Opt. Soc. Am. B, 2014, 31(8): $1777-1785$.

[86] WANG G Q, LIN Y-F, GONG X H, et al. Polarized spectral properties of $\mathrm{Sm}^{3+}: \mathrm{LiYF}_{4}$ crystal. J. Lumin., 2014, 147: 23-26.

[87] WANG G Q, GONG X H, LIN Y F, et al. Polarized spectral properties of $\mathrm{Sm}^{3+}: \mathrm{LiLuF}_{4}$ crystal for visible laser application. Opt. Mater, 2014, 37: 229-234.

[88] MARZAHL D T, METZ P W, KRANKEL C, et al. Spectroscopy and laser operation of $\mathrm{Sm}^{3+}$-doped lithium lutetium tetrafluoride $\left(\mathrm{LiLuF}_{4}\right)$ and strontium hexaaluminate $\left(\mathrm{SrAl}_{12} \mathrm{O}_{19}\right)$. Opt. Express, 2015, 23(16): 21118-211127.

[89] KAZAKOV B N, ORLOV M S, PETROV M V, et al. Induced emission of $\mathrm{Sm}^{3+}$ ions in the visible region of the spectrum. Opt. Spectros, 1979, 47: 676-677.

[90] FARRIES M C, MORKEL P R, TOWNSEND J E. Samarium ${ }^{3+}$ doped glass laser operating at $651 \mathrm{~nm}$. Electron. Lett., 1988, 24(11): 709-711.

[91] KOOPMANN P, LAMRINI S, SCHOLLE K, et al. Holmiumdoped $\mathrm{Lu}_{2} \mathrm{O}_{3}, \mathrm{Y}_{2} \mathrm{O}_{3}$, and $\mathrm{Sc}_{2} \mathrm{O}_{3}$ for lasers above $2.1 \mu \mathrm{m}$. Opt. Ex- press, 2013, 21(3): 3926-3931.

[92] WALSH B M, GREW G W, BARNES N P. Energy levels and intensity parameters of $\mathrm{Ho}^{3+}$ ions in $\mathrm{GdLiF}_{4}, \mathrm{YLiF}_{4}$ and $\mathrm{LuLiF}_{4} . \mathrm{J}$. Phys.-Condens. Mat., 2005, 17(48): 7643-7665.

[93] NOSTRAND M C, PAGE R H, PAYNE S A, et al. Optical properties of $\mathrm{Dy}^{3+}$-and $\mathrm{Nd}^{3+}$-doped $\mathrm{KPb}_{2} \mathrm{Cl}_{5}$. J. Opt. Soc. Am. B, 2001, 18(3): $264-276$.

[94] RADEMAKER K, KRUPKE W F, PAGE R H, et al. Optical properties of $\mathrm{Nd}^{3+}$-and $\mathrm{Tb}^{3+}$-doped $\mathrm{KPb}_{2} \mathrm{Br}_{5}$ and $\mathrm{RbPb}_{2} \mathrm{Br}_{5}$ with low nonradiative decay. J. Opt. Soc. Am. B, 2004, 21(12): 2117 2129.

[95] VORONKO Y K, KAMINSKII A A, OSIKO V V, et al. Stimulated emission of $\mathrm{Ho}^{3+}$ in $\mathrm{CaF}_{2}$ at lambda=551.2 nm. ZhETF Pisma Redaktsiiu, 1965, 1: 5.

[96] CHICKLIS E, NAIMAN C, ESTEROWITZ L, et al. Deep red laser emission in Ho:YLF. IEEE J. Quantum Electron., 1977, 13(11): 893-895.

[97] REICHERT F, MOGLA F, METZ P-W, et al. Prospects of holmiumdoped fluorides as gain media for visible solid state lasers. Opt. Mater. Express, 2015, 5(1): 88-101.

[98] FUNK DAVID-S, EDEN J-GARY. Laser diode-pumped holmiumdoped fluorozirconate glass fiber laser in the green $(\lambda \sim 544-549 \mathrm{~nm})$. IEEE J. Quantum Electron, 2001, 37(8): 980- 992.

[99] GARBUZOV D, KUDRYASHOV I, DUBINSKII M. 110 W(0.9 J) pulsed power from resonantly diode-laser-pumped $1.6-\mu \mathrm{m}$ Er:YAG laser. Appl. Phys. Letter, 2005, 87(12): 121101-1-4.

[100] LI T, BEIL K, KRANKEL C, et al. Efficient high-power continuous wave $\mathrm{Er}^{-\mathrm{Lu}_{2} \mathrm{O}_{3}}$ laser at $2.85 \mu \mathrm{m}$. Opt. Letter, 2012, 37(13): 2568-2570.

[101] JENSEN T, DIENING A, HUBER G, et al. Investigation of diodepumped 2.8- $\mu \mathrm{m}$ Er:LiYF 4 lasers with various doping levels. Opt. Letter, 1996, 21(8): 585-587.

[102] MOGLIA FRANCESCA, MULLER SEBASTIAN, REICHERT FABIAN, et al. Efficient upconversion-pumped continuous wave $\mathrm{Er}^{3+}: \mathrm{LiLuF}_{4}$ lasers. Opt. Mater., 2015, 42: 167-173.

[103] JOHNSON L F, GUGGENHEIM H J. Infrared-pumped visible laser. Appl. Phys. Letter, 1971, 19(2): 44-47.

[104] DANGER T, KOETKE J, BREDE R, et al. Spectroscopy and green upconversion laser emission of $\mathrm{Er}^{3+}$-doped crystals at room temperature. J. Appl. Phys., 1994, 76(3): 1413-1422.

[105] DORENBOS P. The $4 \mathrm{f}^{\mathrm{n}} \leftrightarrow 4 \mathrm{f}^{\mathrm{n}-1} 5 \mathrm{~d}$ transitions of the trivalent lanthanides in halogenides and chalcogenides. J. Lumin., 2000, 91(1/2): 91-106.

[106] TOMA O. Emission regimes of a green Er: $\mathrm{YLiF}_{4}$ Laser. IEEE $J$. Quantum Electron., 2007, 43(7): 519-526.

[107] BREDE R, DANGER T, HEUMANN E, et al. Room-temperature green laser emission of Er:LiYF 4 . Appl. Phys. Lett., 1993, 63(6): 729-730.

[108] 孙家跃, 杜海燕, 胡文祥. 固体发光材料. 北京: 化学工业出 版社, 2003: 89.

[109] DASHKEVICH V I, BAGAYEV S N, ORLOVICH V A, et al. Quasi-continuous wave and continuous wave laser operation of Eu:KGd($\left(\mathrm{WO}_{4}\right)_{2}$ crystal on a ${ }^{5} \mathrm{D}_{0} \rightarrow{ }^{7} \mathrm{~F}_{4}$ transition. Laser Phys. Lett., 2015, 12(1): 015006-1-7.

[110] DEMESH MAXIM, YASUKEVICH ANATOL, KISEL VIKTOR, et al. Spectroscopic properties and continuous-wave deep-red laser operation of Eü ${ }^{3+}$-doped LiYF. Opt. Lett., 2018, 43(10): 2364-2367.

[111] SHI JIAO-JIAO, LIU BIN, WANG QING-GUO, et al. Crystal growth and spectral properties of $\mathrm{Tb}: \mathrm{Lu}_{2} \mathrm{O}_{3}$. Chin. Phys. B, 2018, 27(9): 097801-1-6. 